\title{
The impact of the state of the troposphere on the response to stratospheric heating in a simplified GCM
}

Article

Published Version

Simpson, I. R., Blackburn, M., Haigh, J. D. and Sparrow, S. N. (2010) The impact of the state of the troposphere on the response to stratospheric heating in a simplified GCM. Journal of Climate, 23 (23). pp. 6166-6185. ISSN 1520-0442 doi: https://doi.org/10.1175/2010JCLI3792.1 Available at https://centaur.reading.ac.uk/17549/

It is advisable to refer to the publisher's version if you intend to cite from the work. See Guidance on citing.

Published version at: http://dx.doi.org/10.1175/2010JCLI3792.1

To link to this article DOI: http://dx.doi.org/10.1175/2010JCLI3792.1

Publisher: American Meteorological Society

Publisher statement: (C) Copyright 2010 of the American Meteorological Society. The AMS Copyright Policy is available on the AMS web site at http://www.ametsoc.org

All outputs in CentAUR are protected by Intellectual Property Rights law, including copyright law. Copyright and IPR is retained by the creators or other copyright holders. Terms and conditions for use of this material are defined in the End User Agreement. 


\section{CentAUR}

Central Archive at the University of Reading

Reading's research outputs online 


\title{
The Impact of the State of the Troposphere on the Response to Stratospheric Heating in a Simplified GCM
}

\author{
ISLA R. SIMPSON \\ Department of Physics, Imperial College London, London, United Kingdom, and Department of Physics, \\ University of Toronto, Canada \\ MiCHAEL BLACKBURN \\ National Centre for Atmospheric Science, University of Reading, Reading, United Kingdom \\ JOANNA D. HAIGH \\ Department of Physics, Imperial College London, London, United Kingdom \\ SARAH N. SPARROW \\ Department of Physics, Imperial College London, London, and Department of Meteorology, University of Reading, \\ Reading, United Kingdom
}

(Manuscript received 19 April 2010, in final form 6 August 2010)

\begin{abstract}
Previous studies have made use of simplified general circulation models (sGCMs) to investigate the atmospheric response to various forcings. In particular, several studies have investigated the tropospheric response to changes in stratospheric temperature. This is potentially relevant for many climate forcings. Here the impact of changing the tropospheric climatology on the modeled response to perturbations in stratospheric temperature is investigated by the introduction of topography into the model and altering the tropospheric jet structure.

The results highlight the need for very long integrations so as to determine accurately the magnitude of response. It is found that introducing topography into the model and thus removing the zonally symmetric nature of the model's boundary conditions reduces the magnitude of response to stratospheric heating. However, this reduction is of comparable size to the variability in the magnitude of response between different ensemble members of the same 5000-day experiment.

Investigations into the impact of varying tropospheric jet structure reveal a trend with lower-latitude/ narrower jets having a much larger magnitude response to stratospheric heating than higher-latitude/wider jets. The jet structures that respond more strongly to stratospheric heating also exhibit longer time scale variability in their control run simulations, consistent with the idea that a feedback between the eddies and the mean flow is both responsible for the persistence of the control run variability and important in producing the tropospheric response to stratospheric temperature perturbations.
\end{abstract}

\section{Introduction}

Given the complex nature of the real atmosphere, it is useful to study the atmospheric circulation in a simplified GCM (sGCM) to gain insight into the processes

Corresponding author address: Isla Simpson, Dept. of Physics, University of Toronto, 60 St George St., Toronto ON M5S 1A7, Canada.

E-mail: isla@atmosp.physics.utoronto.ca involved. In recent years many studies have used such models to investigate various aspects of the climate system (e.g., Polvani and Kushner 2002; Kushner and Polvani 2004, 2006; Song and Robinson 2004; Wittman et al. 2004; Haigh et al. 2005, hereafter referred to as HBD05; Son and Lee 2006; Williams 2006; Lorenz and DeWeaver 2007; Gerber and Vallis 2007; Gerber et al. 2008b; Simpson et al. 2009, hereafter referred to as SBH09; Butler et al. 2010). Many of these studies have focused on the tropospheric response to perturbations in 
lower-stratospheric temperature, which is potentially important for many climate forcings such as ozone depletion/recovery (Son et al. 2008b), increased greenhouse gas concentrations (Lorenz and DeWeaver 2007), and solar activity (HBD05).

The present study will focus on the impact of changing certain aspects of the model troposphere on the tropospheric response to stratospheric heating. This work follows from the results of HBD05 and SBH09. In HBD05, it was found that an annular-mode-like response (i.e., an equatorward or poleward shift of the tropospheric midlatitude jets) was produced in response to heating of the model stratosphere. The sign of this response depended on the latitudinal extent of the applied heating perturbation, with low-latitude heating producing a poleward shift of the midlatitude jet and uniform or high-latitude heating producing an equatorward shift of the jet.

SBH09 used spinup ensemble experiments of the same sGCM as HBD05 to investigate the mechanism involved in producing the tropospheric response to stratospheric heating. This demonstrated the important role of changing horizontal eddy momentum fluxes in producing the response. It was found that the altered vertical temperature gradient that accompanied the lowering of the tropopause in response to stratospheric heating weakened the upward flux of eddy activity in the upper-troposphere/ tropopause region. This was accompanied by changes in horizontal eddy momentum flux around the tropopause that were important in both accelerating the zonal wind in the upper troposphere and inducing changes in mean meridional circulation, which acted to accelerate the zonal wind in the lower troposphere. An important feedback was demonstrated: changes in the zonal wind in the troposphere had the effect of refracting the transient wave activity in the troposphere, resulting in altered horizontal eddy momentum flux in the troposphere, which acted to further accelerate the zonal wind anomalies. As the zonal wind anomalies started to grow, a shift in the region of eddy production resulted in a further feedback onto the zonal wind anomalies.

The model used in the above studies will be described in more detail in section 2. It is a Newtonian forced dynamical core; that is, it has a complete representation of the dynamics but physical processes such as moisture and radiation are highly simplified, the mean climate being maintained by relaxation of the temperature field toward a zonally symmetric reference state (Held and Suarez 1994). However, recently some issues with models in this configuration have become apparent (Gerber and Vallis 2007; Gerber et al. 2008b). Although such zonally symmetric temperature relaxation produces fairly realistic climatologies, it is found that the time scale of annular mode variability is often unrealistically long when compared to the real atmosphere (Gerber et al. 2008b). The fluctuation-dissipation theorem (Leith 1975) predicts that the magnitude of the annular-mode-like response to a forcing will be proportional to the projection of the forcing onto that mode, with the constant of proportionality being the time scale of variability in the unforced control run simulation. This has been found to hold, at least qualitatively (Ring and Plumb 2008; Gerber et al. 2008b). It is therefore possible that, if sGCMs are exhibiting unrealistically long time scales of variability, then they may also have unrealistically large responses to forcings such as stratospheric heating.

Gerber and Vallis (2007) showed that time scales of variability were reduced to more realistic values when idealized topography was introduced. Therefore, this study will investigate the impact of introducing idealized topography into the model on the response to stratospheric heating, to determine whether the results of HBD05 and SBH09 hold in the presence of zonally asymmetric boundary conditions, and, if so, what impact the presence of the asymmetry has on the magnitude of response.

Another aspect of the model climatology that may affect the response to a forcing is the structure of the tropospheric midlatitude jet. Indeed, such a sensitivity in the response to polar stratospheric cooling has been demonstrated by Chan and Plumb (2009). They demonstrated that the magnitude of the jet response to the polar stratospheric cooling experiments of Polvani and Kushner (2002) and Kushner and Polvani (2004; 2006) was extremely sensitive to the position of the tropospheric midlatitude jet. They found that the jet of the Polvani-Kushner model existed on the boundary between two distinct regimes: one in which the eddy-driven and subtropical jets were well separated and the other in which they were merged. The troposphere tended to flip between these two regimes and remain in one state or the other for thousands of days. This resulted in an extremely long annular-mode time scale (Gerber and Polvani 2009). Chan and Plumb (2009) demonstrated that the large response to polar cooling in this model was due to the jet being shifted out of this bimodal regime. Altering the tropospheric relaxation temperature profile such as to shift the jet either poleward or equatorward (and so out of the bimodal regime) significantly reduced the annular-mode time scale and the magnitude of response to polar stratospheric cooling.

Gerber and Vallis (2007) have also demonstrated a sensitivity of annular-mode time scales to the structure of the tropospheric jet. They found that weaker equatorto-pole temperature gradients in a simplified GCM produced lower-latitude jets with longer time scales of annular mode variability and vice-versa. By the fluctuation-dissipation theorem it may be expected that 
lower-latitude jets should therefore tend to have a larger annular-mode-like response to a forcing.

Furthermore, some recent evidence suggests that there is a relationship between the annular mode response to climate forcings and the latitude of the climatological jets in more comprehensive GCMs. Son et al. (2010) demonstrated that the poleward shift of the Southern Hemisphere (SH) midlatitude jet in response to ozone depletion in simulations run for the second chemistry climate model validation activity (CCMVal2) models was larger in those models in which the climatological jet was farther equatorward. A similar result was found by Kidston and Gerber (2010) for the poleward shift of the $\mathrm{SH}$ jet associated with climate change in the CMIP3 simulations of the twenty-first century.

Thus, there is a growing body of evidence for an influence of the model jet structure on the model's annular mode time scale and annular-mode-like response to a forcing. Therefore, the effect that changing the tropospheric jet structure has on the response to the stratospheric heating experiments of HBD05 and SBH09 will also be investigated in this study to determine whether the response holds for different jet structures and what impact this has on the magnitude of response.

The structure of the paper is as follows. In section 2 the model and experiments are described. This is then followed by an examination of the impact of topography in section 3 and the impact of changing jet structure in section 4 . The results are discussed and conclusions presented in section 5 .

\section{The model and experiments}

The sGCM used in the following study is the same as that used in HBD05 and SBH09. It is a spectral dynamical core, as described by Hoskins and Simmons (1975), with modification to include the angular-momentumconserving vertical discretization of Simmons and Burridge (1981) while retaining the original sigma coordinate. Triangular truncation at wavenumber 42 is used. There are 15 levels between the surface and $\sigma=$ 0.0185 , with the model levels being $\sigma=0.0185,0.0596$, $0.106,0.152,0.197,0.241,0.287,0.338,0.400,0.477,0.569$, $0.674,0.784,0.887$, and 0.967 . The level spacing is chosen to give good resolution in the tropopause region, which is important for investigations into stratospheretroposphere coupling. Unlike some sGCMs used to investigate stratosphere-troposphere coupling, the model intentionally does not include a fully resolved stratosphere and thus does not exhibit a stratospheric polar vortex. The results are therefore representative of quiescent stratospheric conditions. Gerber et al. (2008a) have demonstrated that, when the stratosphere is more active, the tropospheric annular-mode time scale is increased. This is likely due to sudden stratospheric warmings producing large, long time-scale thermal forcings on the tropospheric jet from the lower stratosphere. The presence of stratospheric variability is unlikely to alter the conclusions here other than necessitating a longer time to separate signal from noise in the response owing to the additional variability in the troposphere coming from the stratospheric variability.

The model has an accurate representation of the large-scale dynamical processes but, in place of the moist and radiative parameterizations of a full GCM, the climate is maintained by Newtonian relaxation of the temperature field toward a zonally symmetric equilibrium state. In the original configuration used in HBD05 and SBH09, this relaxation temperature profile is based on that described by Held and Suarez (1994) and given by

$$
\begin{aligned}
T_{\text {ref }}(\phi, p)= & \max \left\{\left(T_{\text {tpeq }}-\Delta T_{\mathrm{tp}} \sin ^{2} \phi\right),\right. \\
& {\left[T_{o}-\Delta T_{y} \sin ^{2} \phi-\left(\Delta \theta_{\mathrm{eq}} \cos ^{2} \phi\right.\right.} \\
& \left.\left.\left.+\Delta \theta_{\mathrm{pl}} \sin ^{2} \phi\right) \log \left(\frac{p}{p_{0}}\right)\right] \times\left(\frac{p}{p_{o}}\right)^{\kappa}\right\},
\end{aligned}
$$

where $p_{o}$ is the reference surface pressure $(=1000 \mathrm{hPa})$, $T_{\text {tpeq }}$ is the equatorial tropopause temperature, $\Delta T_{\text {tp }}$ is the difference in temperature between the equatorial and polar tropopause, $T_{o}$ is the surface temperature at the equator, $\Delta T_{y}$ is the difference between the equatorial and polar surface temperature, and $\Delta \theta_{\mathrm{eq}}$ and $\Delta \theta_{\mathrm{pl}}$ are the increase in potential temperature with an increase in altitude of one pressure scale height at the equator and poles respectively. The temperature is relaxed toward this profile on a time scale of 40 days for $\sigma<0.7$ (representing radiation and deep moist processes) decreasing to 4 days at the equatorial surface (representing the planetary boundary layer). Boundary layer friction is represented by Rayleigh damping of winds below $\sigma=0.7$ with a time scale of 1 day at the surface. The upper boundary condition is reflective; that is, $\partial \sigma / \partial t=0$ at the model lid. In the original model configuration there is no large-scale zonally asymmetric forcing. Thus planetary waves are weak and are generated only by upscale energy transfer from the dominant synoptic scales. Baroclinic eddies dominate the wave spectrum with peak amplitude at zonal wavenumbers 5-7. These are initiated through a white noise perturbation applied to the surface pressure at the beginning of each equilibrium integration.

\section{a. Introducing topography}

To investigate the impact that zonal asymmetry has on the model response to stratospheric heating, idealized 


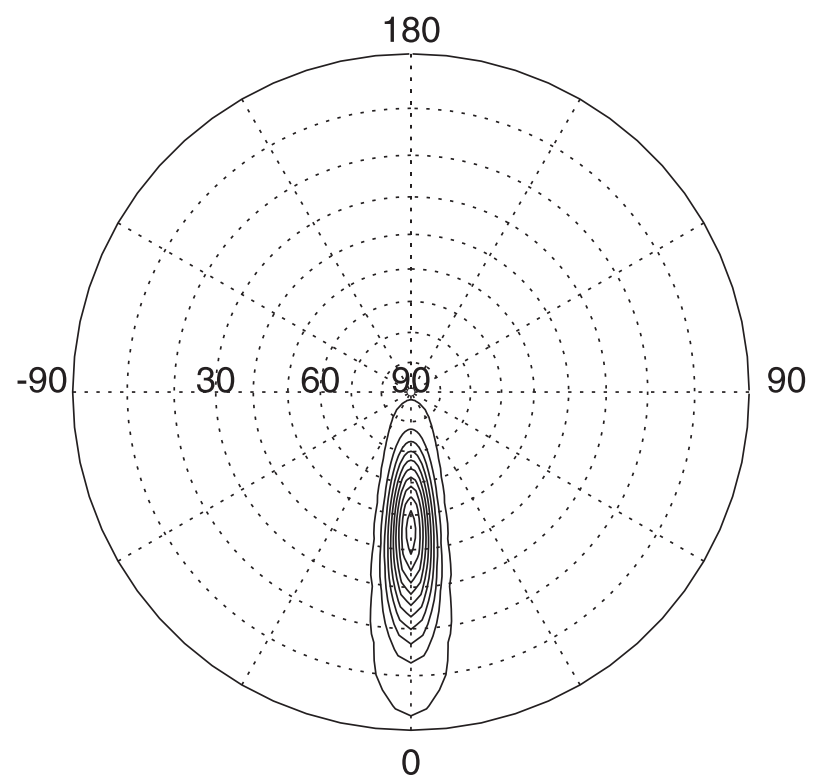

FIG. 1. Topographic height (m), contour interval $200 \mathrm{~m}$.

topography has been added to the Northern Hemisphere $(\mathrm{NH})$ of the model (denoted by run R). An elliptical ridge of 2000-m height, with an eccentricity of 4 and a half-width of $20^{\circ}$ longitude, oriented north-south has been centred at $45^{\circ} \mathrm{N}, 0^{\circ} \mathrm{E}$ (as shown in Fig. 1) to block the jet. It was noted by Gerber and Vallis (2007) that their results were robust for various shapes and heights of idealized topography provided that it was positioned to block the extratropical jet.

\section{b. Changing tropospheric jet structure}

The second set of experiments return to the zonally symmetric configuration of the model and are designed to study the response to stratospheric heating for different zonally symmetric tropospheric jet structures.

In these experiments the jet is altered by modifying the relaxation temperature profile [Eq. (1)] in the troposphere. This has been done for four different tropospheric relaxation temperature profiles. Thus, including the original one, there are five different tropospheric situations that will be denoted by TR1 to TR5. The original Held-Suarez relaxation temperature, shown in Fig. 2a, will be denoted by TR3. The perturbations that are added to this profile to produce the four new tropospheres TR1, TR2, TR4, and TR5 are then shown in Figs. 2b-e.

The pattern in going from TR1 to TR5 is that of increasing midlatitude baroclinicity. TR1 (TR5) reduces (enhances) the equator-to-pole temperature difference by $20 \mathrm{~K}$, by changing the parameters $T_{o}$ and $\Delta T_{y}$ in Eq. (1) to 305 (325) and 40 (80) $\mathrm{K}$ respectively. TR2 and
TR4 have a slightly different relaxation temperature profile given by

$$
\begin{aligned}
T_{\text {ref }}(\phi, p)= & \text { Eq. }(1)+\bar{Q} \cos [2(\phi-\pi / 4)] \\
& \times \sin [4(\phi-\pi / 4)]\left(\frac{p}{p_{o}}\right)^{\kappa},
\end{aligned}
$$

where $\bar{Q}$ is $+2 \mathrm{~K}$ for TR 4 and $-2 \mathrm{~K}$ for TR2. Therefore, TR2 has a reduced midlatitude baroclinicity but with the maximum change in $T_{\text {ref }}$ occurring in the subtropics and subpolar regions. These changes are reversed in TR4. The five different tropospheric $T_{\text {ref }}$ distributions produce different tropospheric jet structures as will be described in section 4 .

\section{c. Stratospheric heating experiments}

The response to stratospheric heating will be examined in each of the tropospheres, described above, with a focus on heating of the equatorial stratosphere (the E5 heating case of HBD05 and SBH09, shading in Fig. 2a). This heating perturbation is introduced by changing the parameters $T_{\text {tpeq }}$ and $\Delta T_{\text {tp }}$ to 205 and $5 \mathrm{~K}$, respectively. For the most part, the equilibrated response to stratospheric heating will be examined. As will be demonstrated in section 3, very long integrations are required to accurately determine the magnitude of response and its uncertainty. Therefore, for each of the experiments an ensemble of 5000-day equilibrium E5 and Control integrations has been performed after an initial spinup of 200 days. For the integrations with topography there are 10 ensemble members. For each of the different tropospheric jet structures there are five ensemble members but, because the model is symmetric about the equator, both hemispheres can be treated as independent samples, providing 10 ensemble members for these integrations also. For two of the tropospheric jet structures (TR2 and TR4) a spinup ensemble experiment has also been performed. Each ensemble consists of 500 integrations of length 150 days, starting from different days of the control run, with the E5 heating switched on at day 0 . Each hemisphere may be treated separately, giving a total sample size of 1000 for each ensemble. All of the above experiments are summarized in Table 1 .

\section{Topography and the need for long runs}

The effect of introducing topography into the $\mathrm{NH}$ of the model has a very similar impact on the tropospheric jet structure (not shown) to that found by Gerber and Vallis (2009). The zonal symmetry of the midlatitude westerly jet is broken, with a region of enhanced zonal wind and vertical wind shear immediately downstream 
(a)

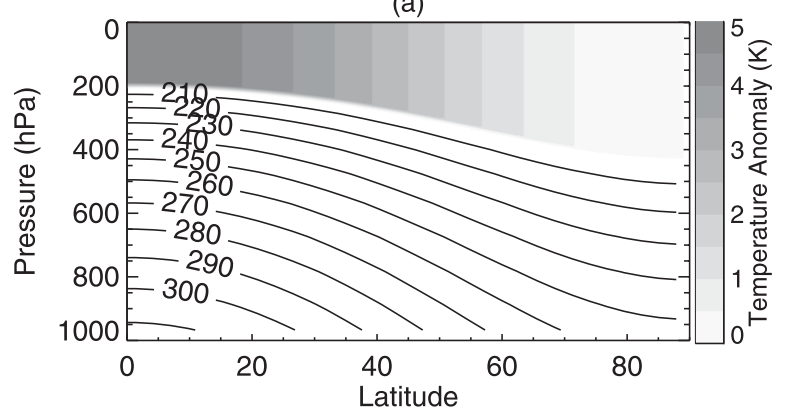

(b)

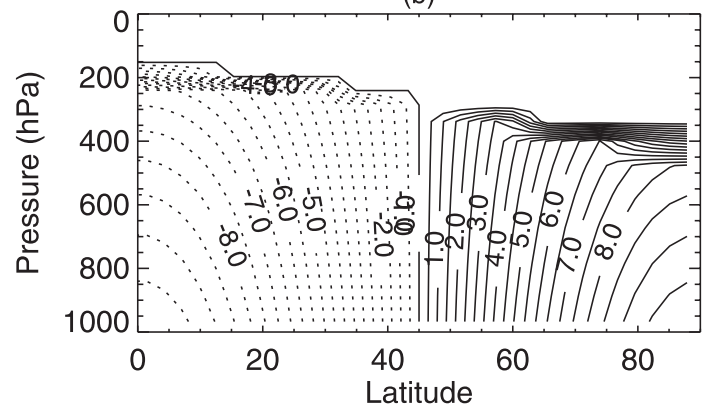

(c)

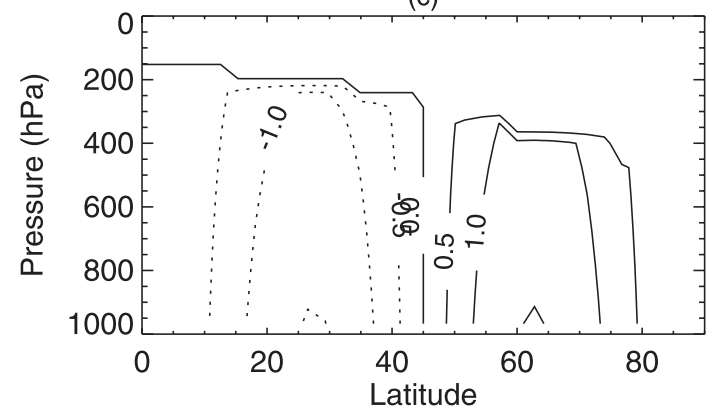

(d)

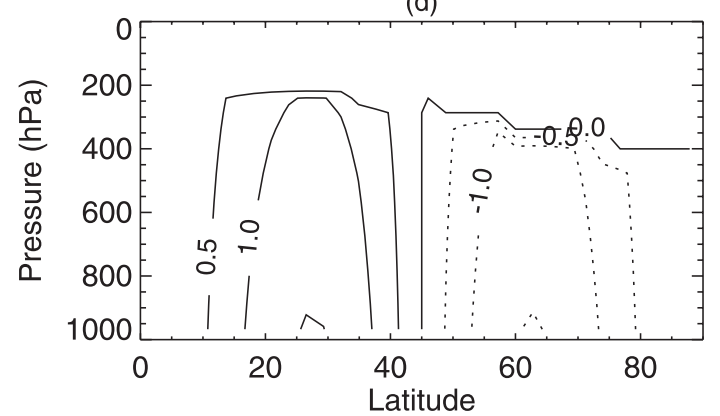

(e)

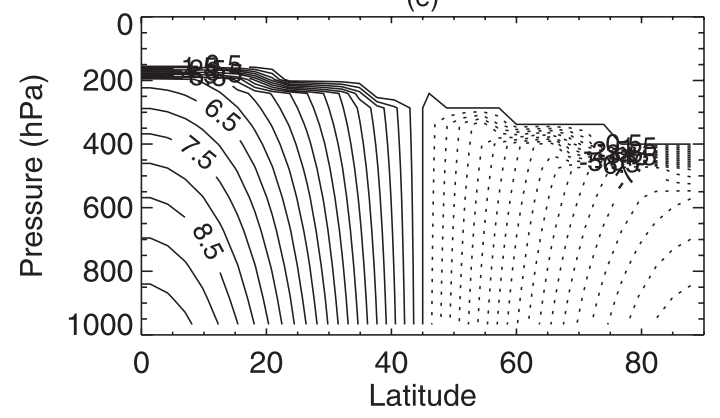

of the topography. Also in agreement with Gerber and Vallis (2009), a stationary wave is generated by the topography, which has the effect of localizing the baroclinicity into several maxima that decrease in magnitude with distance downstream of the topography. Despite the localization in baroclinicity, the eddy kinetic energy remains fairly zonally uniform, and the most prominent zonal variation in the horizontal eddy momentum flux is a maximum occurring at the longitude of the ridge. Gerber and Vallis (2009) suggest that this could be due to shearing of the eddies by the stationary wave generated by the ridge. In the following, the effect of introducing the topography on the response to stratospheric heating will be investigated.

First, however, the issues that these experiments reveal in accurately determining the magnitude of response will be discussed. Figure 3 compares two zonal-mean zonal wind responses to E5 stratospheric heating with topography present in the $\mathrm{NH}$. Each of these is determined from the difference between a 5000-day equilibrated E5 integration and a 5000-day control integration. The two simulations are identical apart from the random number seed used to initiate noise into the model at the beginning of the integration. As there is no topography in the $\mathrm{SH}$, the response in this hemisphere is equivalent to that of HBD05 and SBH09.

In each of the hemispheres of Figs. $3 a$ and $3 b$ a qualitatively similar pattern of response is produced, consisting of a poleward shift of the midlatitude jet. In the first experiment (Fig. 3a), the magnitude of response is considerably reduced in the $\mathrm{NH}$ where the topography is present. This may be what is expected from the results of Gerber and Vallis (2007) regarding the time scale of control run variability and the fluctuation-dissipation theorem. However, in the second experiment (Fig. 3b) the magnitude of response is completely different. Both hemispheres have a reduced magnitude compared to the SH of Fig. 3a, but the NH now has a larger response than the SH. These are two identical experiments, with the exception of the initial random perturbation used to introduce noise into the model, but different conclusions could be drawn from them.

This example demonstrates that 5000-day integrations are insufficient to determine accurately the magnitude of response. This has very little bearing on the results of

FIG. 2. Relaxation temperature profiles $T_{\text {ref }}$ for the various different tropospheric states: (a) The Held-Suarez relaxation temperature profile (TR3) (contours, interval $10 \mathrm{~K}$ ) and the E5 heating perturbation (shading, contour interval $0.5 \mathrm{~K})$; (b)-(e) the perturbation that is added onto (a) to give tropospheres TR1, TR2, TR4, and TR5, respectively (contour interval $0.5 \mathrm{~K}$ ). 
TABLE 1. Summary of model runs.

\begin{tabular}{|c|c|c|c|}
\hline \multicolumn{4}{|c|}{ Zonally asymmetric boundary conditions } \\
\hline Name & Type & Length & Description \\
\hline $\mathrm{R}$ & Control and E5 & $10 \times 5000$ days & Eq. (1) $T_{\text {ref }}$, with topography in NH, $T_{o}=315, \Delta T_{y}=60$ \\
\hline TR1 & Control and E5 & $2 \times 5 \times 5000$ days & Eq. (1) $T_{\text {ref }}, T_{o}=305, \Delta T_{y}=40$ \\
\hline TR2 & Control and E5 & $2 \times 5 \times 5000$ days & Eq. (2) $T_{\text {ref }}, T_{o}=315, \Delta T_{y}=60, \bar{Q}=-2 \mathrm{~K}$ \\
\hline TR2 & E5 spinup & $2 \times 500 \times 150$ days & - \\
\hline TR3 & Control and E5 & $2 \times 5 \times 5000$ days & Eq. (1) $T_{\text {ref }}, T_{o}=315, \Delta T_{y}=60$ \\
\hline TR4 & Control and E5 & $2 \times 5 \times 5000$ days & Eq. (2) $T_{\text {ref }}, T_{o}=315, \Delta T_{y}=60, \bar{Q}=2 \mathrm{~K}$ \\
\hline TR4 & E5 spinup & $2 \times 500 \times 150$ days & - \\
\hline TR5 & Control and E5 & $2 \times 5 \times 5000$ days & Eq. (1) $T_{\text {ref }}, T_{o}=325, \Delta T_{y}=80$ \\
\hline
\end{tabular}

HBD05 and SBH09, where qualitative patterns of response are examined because these are robust. It demonstrates, however, that very long integrations or ensembles are required when examining responses such as this in a quantitative manner.

Examination of time series of zonal-mean zonal wind for the control and equilibrium integrations reveals that the jet undergoes regime shifts. For a large proportion of the integrations the variability of the jet is characterized by poleward propagating anomalies and relatively short time-scale annular mode variability. There are, however, occasions when the jet remains in an anomalously poleward or equatorward position for an extended period. This can be seen in Fig. 4, which shows a 600-day sample of zonal wind (anomalies from the time mean) from the $\mathrm{SH}$ of one of the $\mathrm{R}$ runs. The second half of this time series is characterized by poleward propagating anomalies, whereas in the first half the jet is positioned anomalously equatorward for around 200 days. Such anomalies can last for several hundred days. These two types of variability have been identified by Son and Lee (2006) and will be discussed further in section 4 . These are present in both the hemisphere with and without topography. Thus, although on average the time scale of variability may be fairly short (e.g., 40 days), the presence of these occasional long-time-scale anomalies leads to large uncertainties in a forced response that is of relatively small magnitude compared to the natural variability occurring within both the control and experiment equilibrium runs.

The impact of topography on the response to stratospheric heating is now examined in an ensemble of 10 runs, each of 5000 days, to allow a more accurate determination of the magnitude of response and its uncertainty in the presence of natural variability.

The ensemble-mean zonal wind response to E5 stratospheric heating with topography in the $\mathrm{NH}$ and its standard deviation are shown in Fig. 5. Comparison of the $\mathrm{SH}$ and $\mathrm{NH}$ shows that introducing idealized topography does not change the qualitative pattern of response of HBD05 and SBH09. Comparison of the stationary and transient eddy momentum flux (not shown) demonstrates that, even when topography is introduced, the transient eddy momentum flux remains dominant in the response to stratospheric heating.

The ensemble mean response to stratospheric heating does show asymmetry between the hemispheres, with the magnitude of response in the $\mathrm{NH}$ being about half that in the SH. This difference, however, is smaller than the magnitude of variability between 5000-day means, as can be seen by the large standard deviation in Fig. 5 b.

A clearer sense of the variability is given in Fig. 6a, which shows the zonal-mean zonal wind response at two
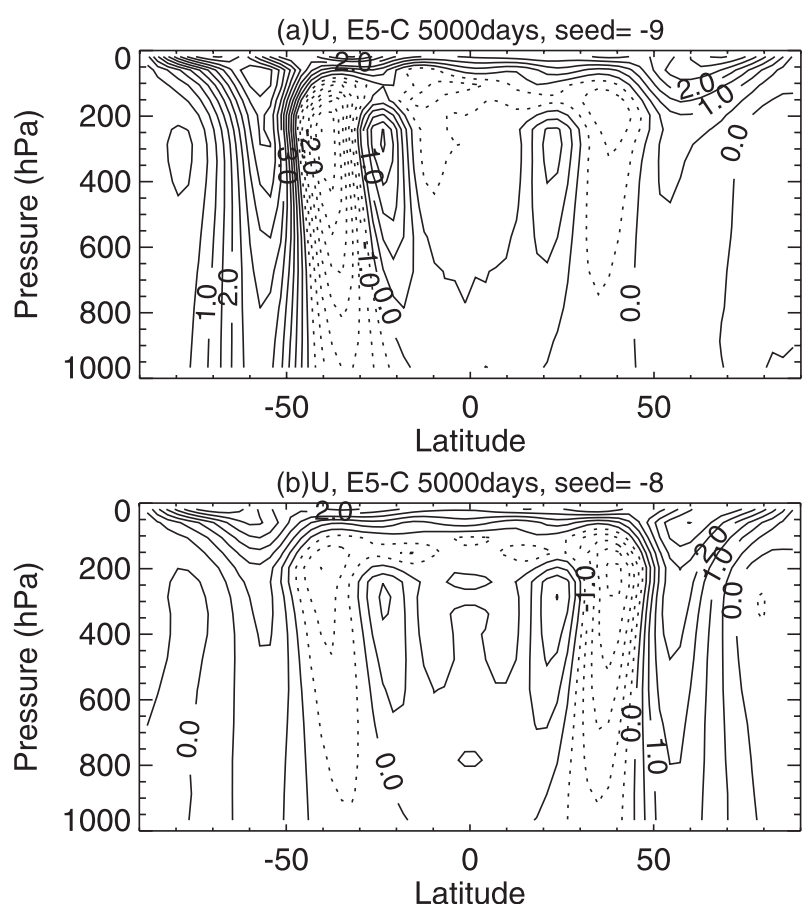

FIG. 3. Zonal-mean zonal wind $\left(\mathrm{m} \mathrm{s}^{-1}\right)$ response to E5 stratospheric heating for two 5000-day equilibrium runs with topography in the $\mathrm{NH}$, which differ only in the initial random number seed used to initiate noise into the model (contour interval $0.5 \mathrm{~m} \mathrm{~s}^{-1}$ ). 


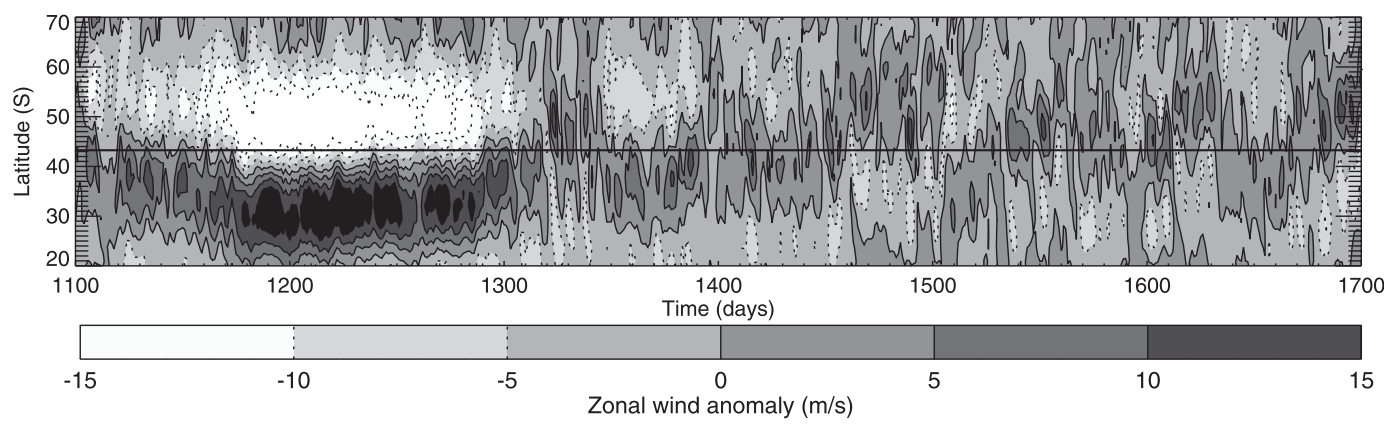

FIG. 4. Time series of zonal-mean zonal wind anomalies from the time mean at the 286-hPa level for the SH of one of the $\mathrm{R}$ runs.

points on the $286-\mathrm{hPa}$ level: $54^{\circ}$ and $37^{\circ}$ latitude in both $\mathrm{NH}$ and $\mathrm{SH}$. These are around the maximum zonal wind increase on the poleward side of the jet and the maximum zonal wind decrease on the equatorward side of the jet, respectively. Each individual ensemble member together with the ensemble mean and $95 \%$ confidence interval are shown. This demonstrates that there is a large spread in the magnitude of response over the 10 different 5000-day ensemble members. In the extreme cases, there can be up to an order of magnitude difference in the size of the peak wind anomaly. Nevertheless, the ensemble means suggest that there is a reduction in the magnitude of response of between one-half and onethird when topography is present. However, this is barely significant and, given the large spread between the ensemble members, it is difficult to assign a value to this reduction with certainty. ${ }^{1}$

Another common measure of the magnitude of a response is the projection of that response onto the dominant modes of control run variability. The first and second empirical orthogonal functions (EOFs) account for most of the variability in the zonal-mean zonal wind with the first EOF representing a poleward shift of the midlatitude jet and the second EOF representing a weakening/strengthening and broadening/narrowing of the jet (see Fig. 1 of Sparrow et al. 2009). Figure 6b shows the projection of the zonal wind response onto the first and second EOFs (calculated following Baldwin et al. 2009) of the zonal-mean zonal wind variability of the $\mathrm{NH}$ and the $\mathrm{SH}$ of the $\mathrm{R}$ runs. The response predominantly projects onto EOF1, which is expected given that the response is primarily a poleward shift of the jet. Much like the zonal wind anomalies in Fig. 6a, there is

\footnotetext{
${ }^{1}$ The influence of topography has only been investigated for the equatorial heating case. The other heating cases of HBD05 and SBH09 have not been investigated owing to the need for these extremely long runs. However, it is likely that the same conclusions will hold for these heating cases.
}

a large amount of variability in the response projections. The projection of the response is larger in the $\mathrm{SH}$ but, given the large amount of variability between ensemble members, the significance is low.

For comparison with the results of Gerber et al. (2008b), the decorrelation time scale of the model's annular mode has been calculated. This is done by projecting the zonal wind anomaly from the time mean onto the first EOF and then determining the mean $e$-folding time scale of the autocorrelation of that projection (this is the same method as used by, e.g., Chan and Plumb 2009).

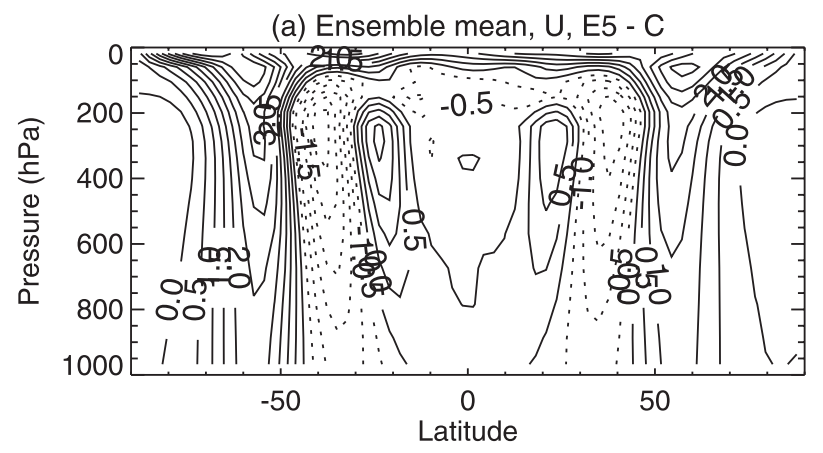

(b) Standard deviation

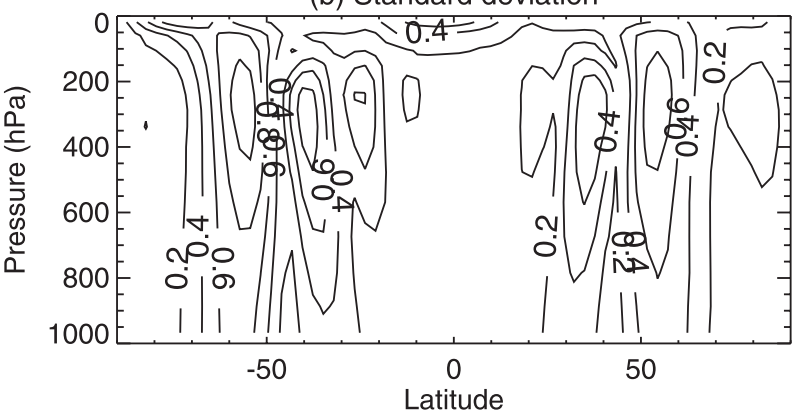

FIG. 5. (a) Ensemble mean zonal-mean zonal wind response $\left(\mathrm{m} \mathrm{s}^{-1}\right)$ to $\mathrm{E} 5$ stratospheric heating with topography in the $\mathrm{NH}$ (contour interval $0.5 \mathrm{~m} \mathrm{~s}^{-1}$ ) and (b) standard deviation as calculated from the 10 different ensemble members (contour interval $0.2 \mathrm{~m} \mathrm{~s}^{-1}$ ). 
(a) $+=(54 \mathrm{deg}, 286 \mathrm{hPa}),{ }^{*}=(37 \mathrm{deg}, 286 \mathrm{hPa})$

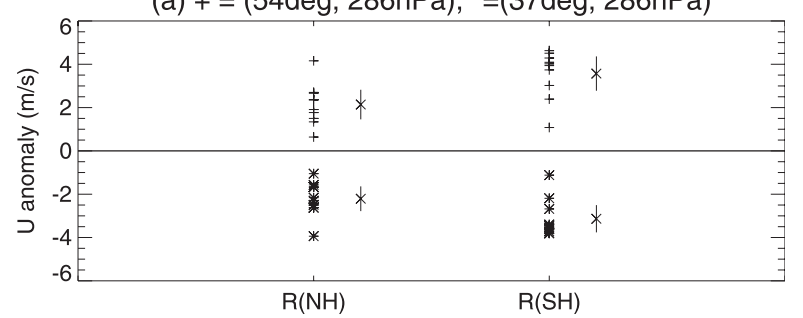

(b) Projection of $U$ response onto EOFs 1 and 2

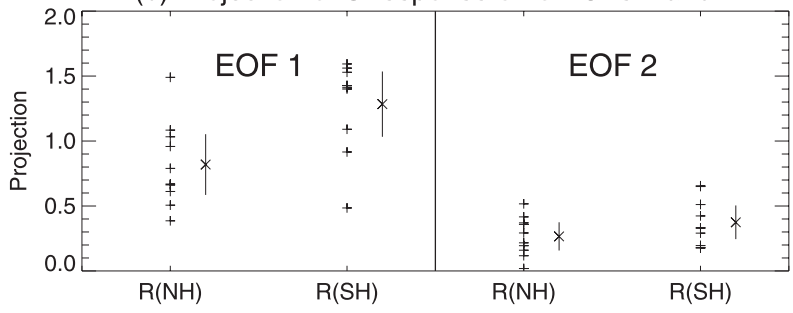

(c) Decorrelation timescale

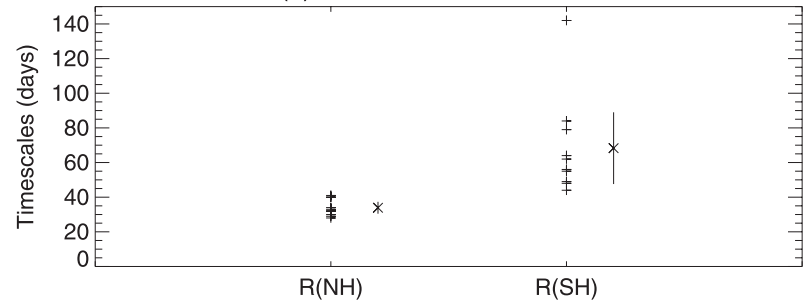

FIG. 6. (a) Zonal-mean zonal wind response to E5 stratospheric heating at the $286-\mathrm{hPa}$ level and $37^{\circ}$ and $57^{\circ}$ latitude for topography in the NH. (b) Projection of the zonal wind response onto EOF1 and EOF2 for the NH and the SH. (c) Decorrelation time scale of the first EOF of zonal mean zonal wind variability. Each figure shows values for individual ensemble members $(+)$ with the mean $(\times)$ and $95 \%$ confidence interval (vertical line) plotted to the right of each.

Figure $6 \mathrm{c}$ shows the time scales for each individual ensemble member together with the mean and $95 \%$ confidence interval. It is apparent that there is a clear separation in the time scale between the $\mathrm{NH}$ and the $\mathrm{SH}$ of the run, both in the ensemble mean value and in its spread. The shorter time scale in the presence of the topography is more representative of estimates for the earth's atmosphere [ 10-20 days (Baldwin et al. 2003)]. There is also a significant reduction in the spread of the time scale between different ensemble members. The spread in time scale is much larger with zonally symmetric forcing, with values in individual integrations ranging from $\sim 45$ to (an extreme of) $\sim 140$ days. A single 5000-day experiment could have produced either similar time scales for both the $\mathrm{NH}$ and the $\mathrm{SH}$ or, alternatively, a time-scale reduction by a factor of 3-4 due to topography. This, again, highlights the need for long integrations, not only in determining the magnitude of response but in characterizing the control run variability.
Focusing on the ensemble mean, there is a clear impact of the topography on the time scale of variability, reducing the ensemble mean time scale in the $\mathrm{NH}$ to about half that of the $\mathrm{SH}$. This is consistent with the results of Gerber and Vallis (2007). The mean magnitude of response in the $\mathrm{NH}$ has also been reduced to about half that of the $\mathrm{SH}$, which is what is expected from the fluctuation-dissipation theorem. Given the spread in the magnitudes of response, however, it is difficult to say this with certainty. It appears that topography is reducing the magnitude of response slightly but, given the large uncertainties, it is difficult to assign a magnitude to this reduction.

It is interesting to note that, although the time scale in the presence of topography appears to be much more constrained (Fig. 6b), the magnitude of response to stratospheric heating is not (Fig. 6a). That is, although the hemisphere with topography appears to be rather well constrained in terms of its variability, there is still a large uncertainty in the magnitude of response.

The original aim of these experiments was to determine the impact of introducing zonal asymmetry in the form of idealized topography on the magnitude of response to stratospheric heating. An important conclusion to be taken from the results, however, is the need for extremely long integrations in idealized models both to accurately characterize the variability and to determine the magnitude of response to a forcing. This arises because, although the dominant variability is of rather short time scale, there is an underlying low frequency variability with regime shifts in which the jet may remain at an anomalously high or low latitude for an extended period of time. The model's annular variability therefore exhibits a wide range of time scales (Sparrow et al. 2009). Long integrations are required both to accurately characterize the control run variability and to determine the response to a forcing when that response is comparable to, or smaller than, the intrinsic variability. These experiments have only been performed with one model, but the presence of multiple modes of variability in other sGCMs (e.g., Son and Lee 2006) suggests that this is a feature of all models with a similar Newtonian forced configuration.

\section{The effect of varying tropospheric jet structure}

The impact of changing the structure of the tropospheric jet on the response to stratospheric heating in the zonally symmetric model configuration is now investigated. The previous studies of HBD05 and SBH09 have focused on the response with the model in the configuration of Held and Suarez (1994). Gerber and Vallis (2007) and Son and Lee (2006), however, have 
shown that changing the tropospheric relaxation temperature structure of a sGCM causes a change in the natural variability of the midlatitude westerlies. This is likely to have an impact on the response to stratospheric heating, as predicted by the fluctuation-dissipation theorem.

Here, as described in section $2 b$, four new tropospheres have been created by altering the tropospheric relaxation temperature distribution $\left(T_{\text {ref }}\right)$. This section begins with a discussion of the control run states of each troposphere. Then the impact of changing the tropospheric state on the equilibrated response to E5 heating will be shown, and some preliminary evidence will be presented for the mechanism leading to the variations in response.

The lhs of Fig. 7 shows the control run zonal-mean zonal wind for each of the tropospheres TR1 to TR5. In going from TR1 to TR5, there is an increase in the midlatitude temperature gradient, reflecting the increasing baroclinicity in the reference state. Associated with this, there is an increase in eddy activity including increasing poleward eddy heat flux in the midlatitude lower troposphere and increasing poleward eddy momentum flux in the midlatitude upper troposphere. This can be seen in the lhs of Fig. 8, which shows the control run Eliassen-Palm (E-P) flux (scaled as in Edmon et al. 1980) for each of the tropospheres. This results in a change in the structure of the climatological jet. In going from TR1 to TR5, the jet becomes stronger and broader and the eddy-driven midlatitude jet becomes located at higher latitudes, with the midlatitude and subtropical jets becoming increasingly separated. Stronger surface winds (easterlies at low latitudes and westerlies at higher latitudes) arise from stronger poleward momentum flux (equatorward E-P flux).

\section{a. The response to E5 stratospheric heating}

The response to E5 stratospheric heating is shown in the right-hand columns of Figs. 7 and 8 for zonal-mean zonal wind and E-P flux, respectively, for each of the tropospheres. The pattern of response for each troposphere is qualitatively similar, each showing the familiar patterns of TR3, which have been discussed extensively in HBD05 and SBH09. However, there is a large difference in the magnitude of response between the tropospheres, with TR1 having a much stronger response and TR5 having a much weaker response than the original TR3 experiment. There is almost an order of magnitude difference in the zonal wind response between TR1 and TR5 with, for example, a peak zonal wind increase on the poleward side of the jet at $\sim 250 \mathrm{hPa}$ being around $10 \mathrm{~m} \mathrm{~s}^{-1}$ in TR1 compared to around $1.5 \mathrm{~m} \mathrm{~s}^{-1}$ in TR5.
As mentioned in the introduction, in certain configurations simplified GCM climates lie on the border between two regimes: one where the jet is at much higher latitude than the other with $\sim 10^{\circ}$ latitude difference in the two preferred locations (Gerber and Polvani 2009; Chan and Plumb 2009); this constitutes a bimodal distribution of the latitude of maximum surface westerlies. Chan and Plumb (2009) demonstrated that this bimodality can account for the very large response to polar stratospheric cooling in the studies of Polvani and Kushner (2002) and Kushner and Polvani (2004). They showed that shifting the jet poleward or equatorward brought the jet out of this bimodal regime and reduced both the time scale and the magnitude of response to polar stratospheric cooling.

For each of the tropospheric situations presented here, although there are large fluctuations around the time mean of the jet maximum, as seen in Fig. 4, these result in only small fluctuations in the position of maximum surface westerlies and represent fluctuations around a mean jet position rather than a bimodal distribution of jet location. Moreover, in the spinup ensembles to be presented in the following section, it is clear that the zonal wind anomalies change in a continuous manner rather than jumping from one regime to the other. The results therefore differ from Chan and Plumb (2009) in that none of the tropospheric situations exist in a bimodal regime. Rather, there appears to be a quasi-linear relationship between the latitude of the jet and the time scale of variability and the magnitude of response to stratospheric heating.

It is perhaps surprising, given the importance of eddy fluxes in producing the tropospheric response (SBH09), that there is a stronger response for the lower-latitude jets despite the weaker eddies (and eddy fluxes) in their control run climates.

The difficulty of accurately determining the magnitude of response has already been demonstrated, and it must be stressed that these differences between the ensemble mean responses of the different tropospheres occur concurrently with a significant amount of variability between individual ensemble members for each troposphere. This is demonstrated in Fig. 9, which shows various diagnostics of the magnitude of response. Figure 9a takes the average magnitude of the zonal wind over all latitudes and pressures as a measure of the response magnitude. This is shown for each individual ensemble member together with the mean and $95 \%$ confidence interval. Despite the ensemble spread, the ensemble means exhibit a clear signal of varying response magnitude with different tropospheric jet structure that is statistically robust (e.g., compare the ensemble means and $95 \%$ confidence intervals of Fig. 9a). This is further 
U, TR1 (C)

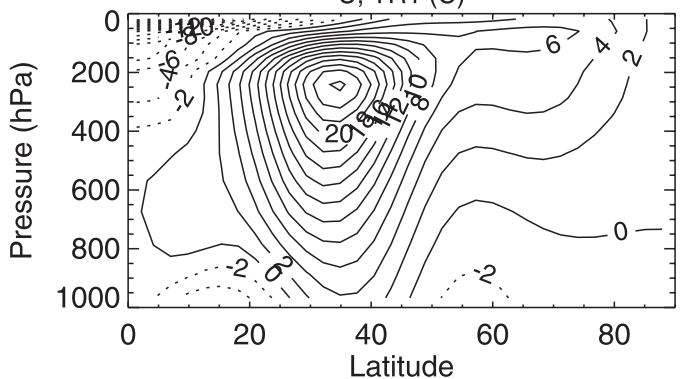

$\mathrm{U}, \mathrm{TR} 2(\mathrm{C})$

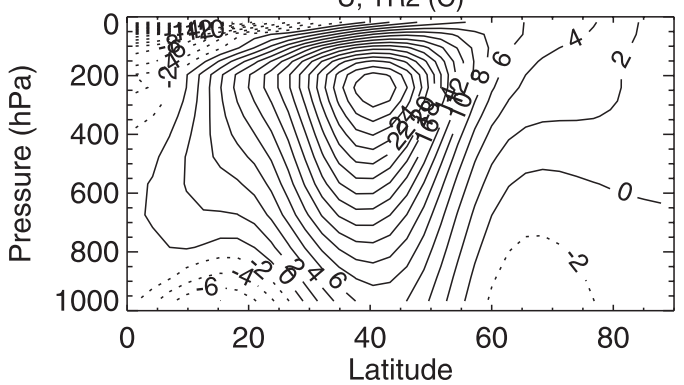

$\mathrm{U}, \mathrm{TR} 3(\mathrm{C})$

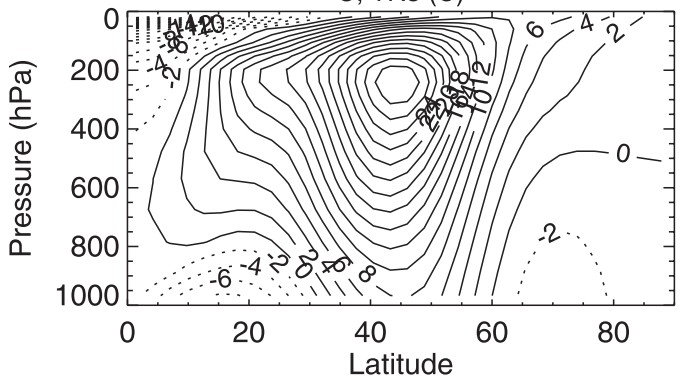

U, TR4 (C)

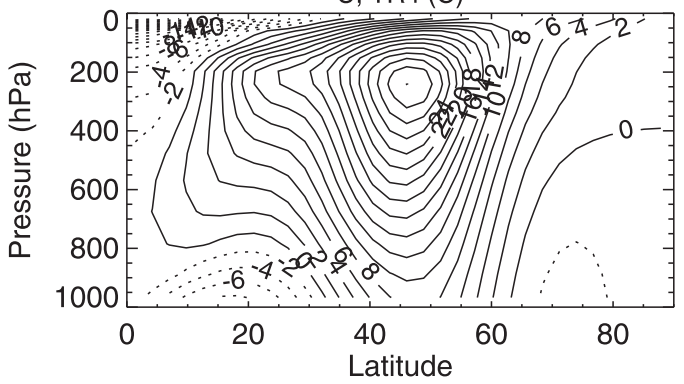

$\mathrm{U}, \mathrm{TR} 5$ (C)

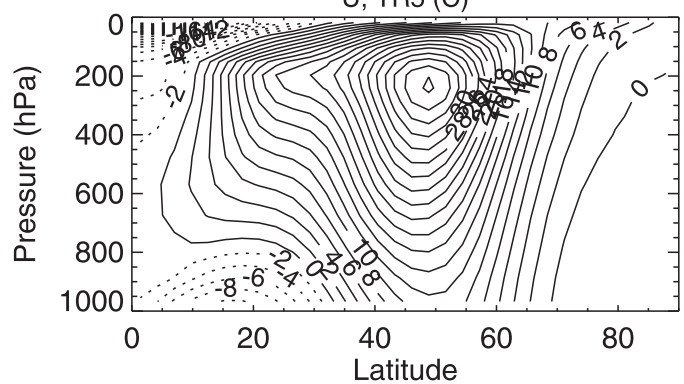

U, TR1 (E5-C)

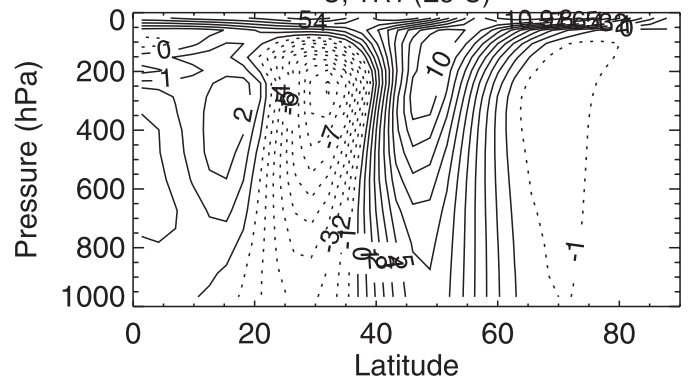

$\mathrm{U}, \mathrm{TR} 2$ (E5-C)

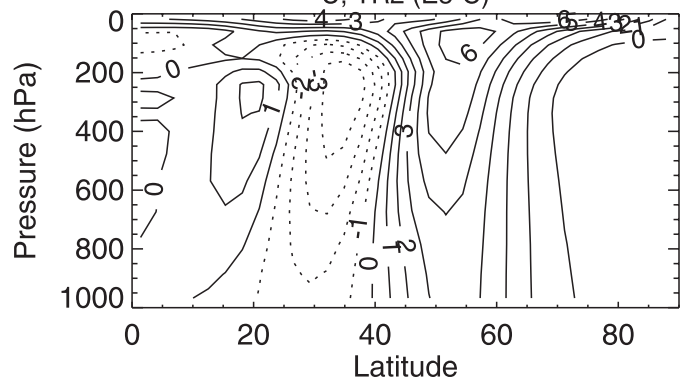

U, TR3 (E5-C)

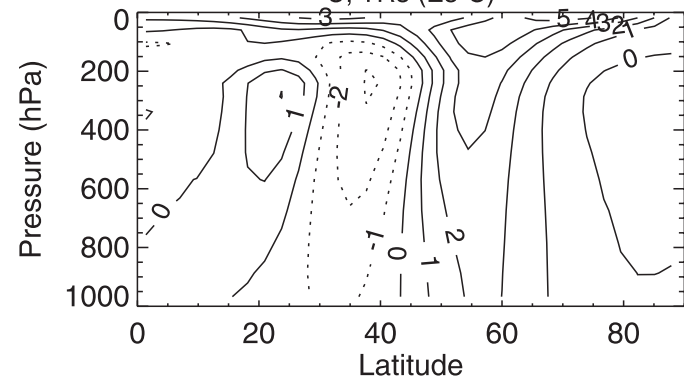

U, TR4 (E5-C)
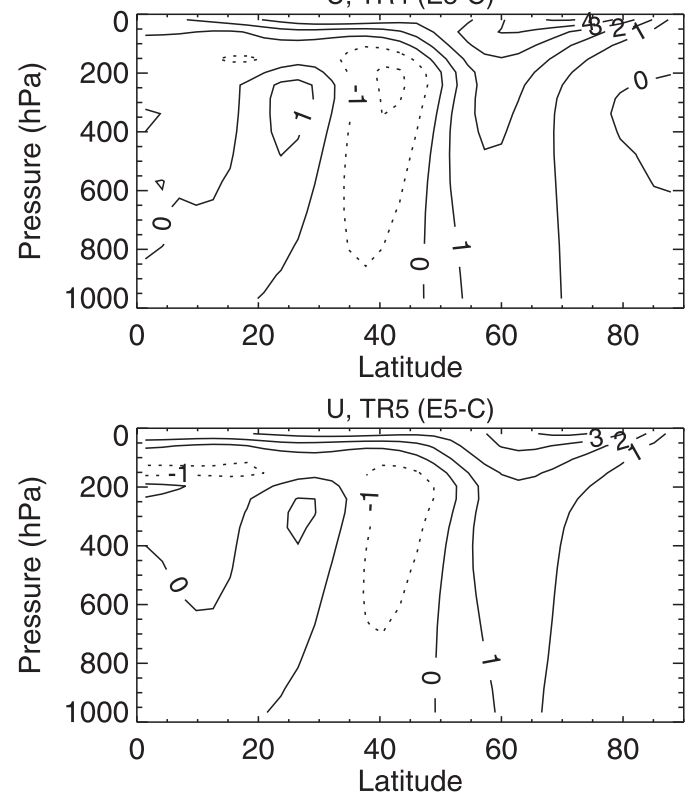

FIG. 7. (left) Zonal-mean zonal wind $\left(\mathrm{m} \mathrm{s}^{-1}\right)$ of the ensemble mean control runs of tropospheres (top) TR1 to (bottom) TR5 (contour interval $2 \mathrm{~m} \mathrm{~s}^{-1}$ ), and (right) ensemble mean zonal-mean zonal wind response $\left(\mathrm{m} \mathrm{s}^{-1}\right)$ to E5 stratospheric heating for (top) TR1 to (bottom) TR5 (contour interval $1 \mathrm{~m} \mathrm{~s}^{-1}$ ). 

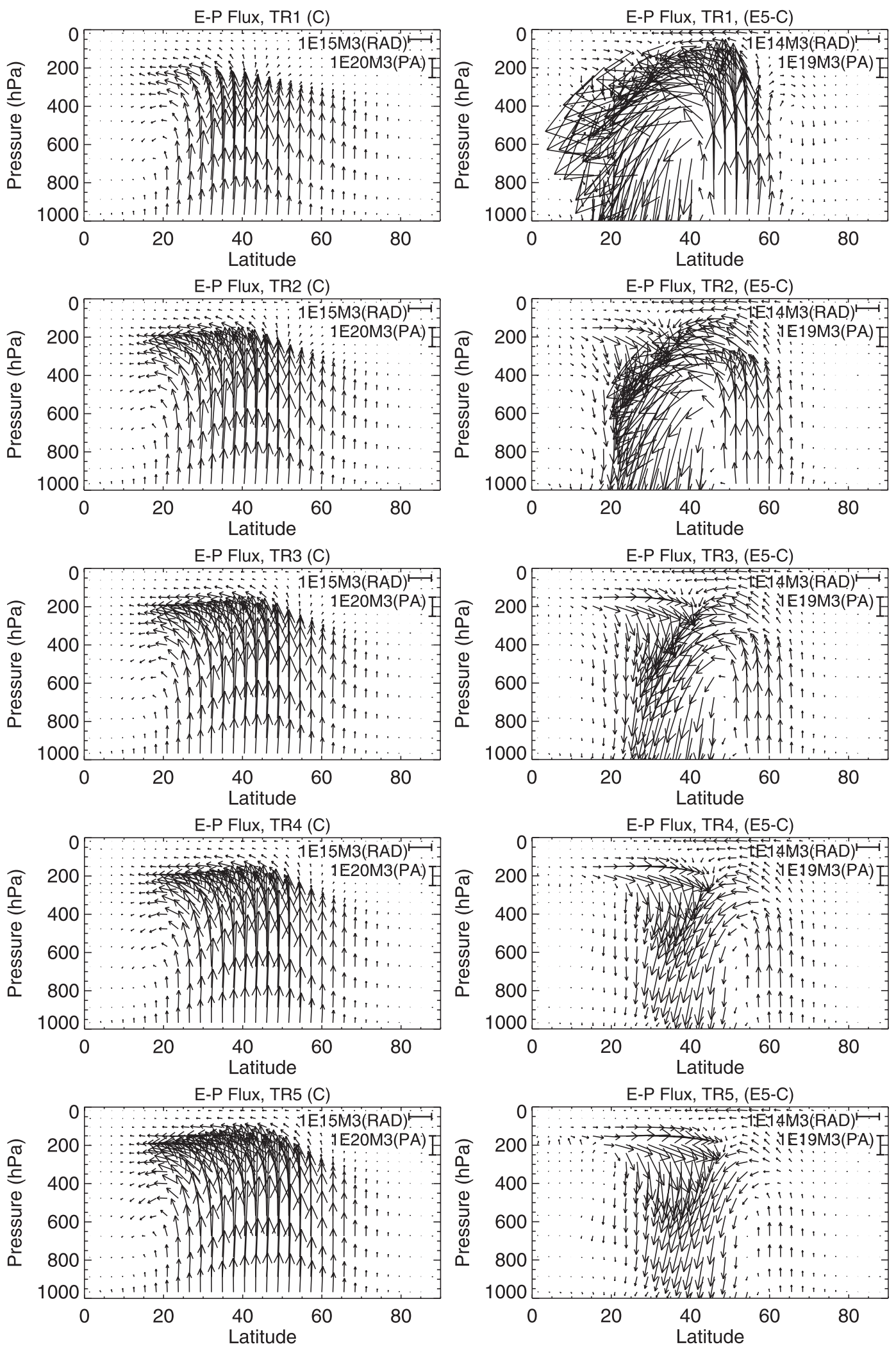

FIG. 8. (left) Eliassen-Palm flux for the ensemble mean control runs and (right) ensemble mean E-P flux response to E5 stratospheric heating for tropospheres (top) TR1 to (bottom) TR5. 
(a) Magnitude of response

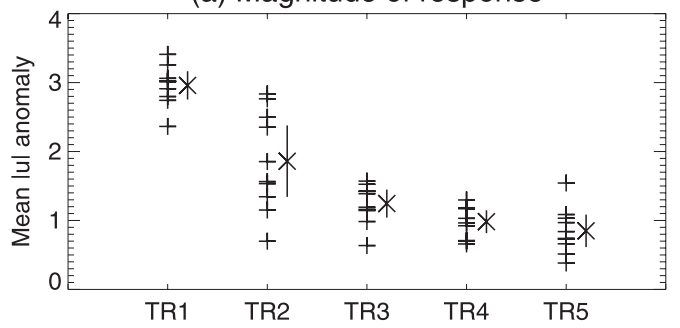

(c) Projection of $U$ anomaly onto EOF1

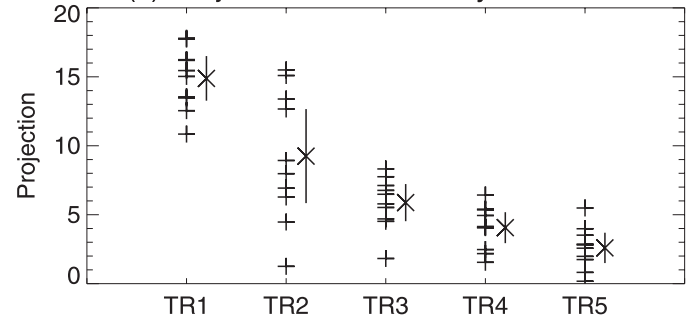

(b) Decorrelation timescales

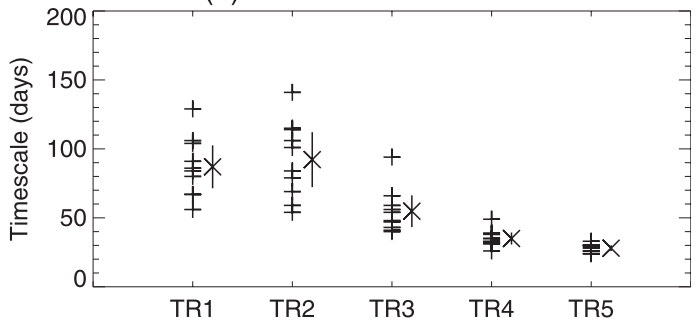

(d) Projection of U anomaly onto EOF2

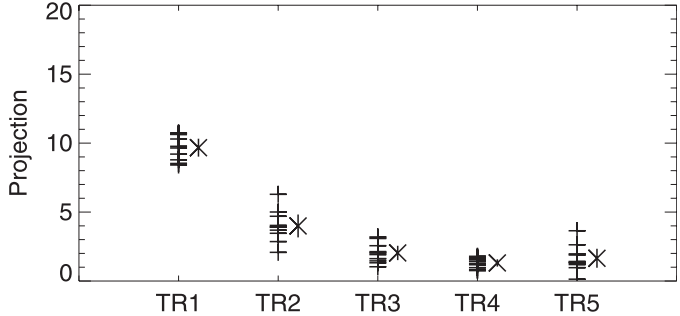

FIG. 9. (a) Mean magnitude of zonal-mean zonal wind response to E5 stratospheric heating (average over the latitude-pressure plane), (b) decorrelation time scale of the variability of the first EOF of zonal-mean zonal wind variability, (c) projection of the zonal wind response onto EOF1, and (d) projection of the zonal wind response onto EOF2. All plots show values for each individual ensemble member $(+)$ together with the ensemble mean $(\times)$ and $95 \%$ confidence interval (vertical line) plotted to the right of each.

verified by the difference in spinup evolution of TR2 and TR4 to be presented in the following section. A similar variation can also be seen in the projection of the zonal wind anomalies onto the first and second EOFs in Figs. 9c and 9d. Again, the response predominantly projects onto EOF1 with a lesser projection onto EOF2. In both these projections there is a clear trend that lower-latitude jets have a response to E5 heating that is larger and thus projects more strongly onto the dominant modes of variability. It should be noted that the first and second EOFs are very similar for the different tropospheres, with EOF1 representing a shift around the jet center and EOF2 a strengthening/narrowing and weakening/broadening of the jet. From this it is clear that lower-latitude jets, which are relatively weak and narrow, have a much larger response than higher-latitude jets that are relatively strong and broad. ${ }^{2}$

Examination of equilibrated responses such as these carries with it the usual difficulty of separating cause from effect because the eddies and mean flow are highly coupled. Comparing the rhs of Figs. 7 and 8 shows that the tropospheres that have a larger magnitude of response in zonal wind also have larger anomalies in E-P

\footnotetext{
${ }^{2}$ A similar variation is found in the response to polar stratospheric heating, although it is less dramatic. Thus, the primary cause of this sensitivity of response to changing jet structure does not appear to be related to the proximity of the midlatitude jet to the imposed stratospheric heating.
}

flux, consistent with the ideas of SBH09 regarding the mechanism of production of the tropospheric response. The anomalous meridional gradient in zonal wind across the jet center alters the refraction of the eddies and changes their momentum fluxes, resulting in a positive feedback onto the zonal wind anomalies (see SBH09). Another component of the feedback is that in the region of increased vertical wind shear there is an increase in the source of baroclinic activity and vice-versa (analogous to the self-maintaining jet mechanism of Robinson 2000). It is clear that the lower-latitude/narrower jets have much larger E-P flux anomalies and, therefore, E-P flux convergence/divergence anomalies that act to accelerate the zonal flow. These E-P flux anomalies, however, are themselves due to the zonal wind anomalies, so the magnitude of the change in E-P flux and the change in zonal wind are intrinsically linked: it cannot be said that one causes the other in these equilibrated experiments.

Rather, there is some aspect of the lower-latitude/ narrower jets that causes the feedback between the eddies and the mean flow to be stronger, allowing the zonal wind and eddy momentum flux anomalies to grow larger together, resulting in an equilibrium response that is much larger than that for higher-latitude/wider jets. It is not obvious why this should be the case, given the importance of eddy fluxes in producing the response and the fact that lower-latitude/narrower jets are associated with weaker climatological eddy fluxes. 


\section{b. Control run variability}

Given that other studies have demonstrated that the simulated response of the tropospheric midlatitude jets to various forcings is closely related to the natural variability in the unforced control run simulations (Gerber and Vallis 2007; Ring and Plumb 2008), it is instructive to examine the control run variability of the TR1-TR5 simulations.

Figure 10 presents one-point correlation maps for the wind anomalies of each troposphere, at the $286-\mathrm{hPa}$ level, as a function of latitude and time lag, with the base latitude chosen to be the latitude of maximum variability on the equatorward side of the jet maximum (similar results are produced for any base latitude within the region of dipole variability around the jet center). The one-point correlation maps demonstrate that TR5 has short-time-scale zonal wind anomalies with a tendency for poleward propagation. The variability time scale increases going from TR5 to TR1 and the presence of the poleward propagation disappears.

A similar variation is also apparent in the decorrelation time scale presented in Fig. 9b for each ensemble member together with the ensemble mean and $95 \%$ confidence interval. There is a general trend of an increase in the decorrelation time scale in going from TR5 to TR1. Here TR4 and TR5 have considerably shorter time scales of variability than TR1 and TR2, while TR3 has an intermediate time scale. However, as the time scale increases, so does the ensemble spread and hence the uncertainty in the estimated value.

It is interesting to note that there is a consistent pattern in all equilibrium runs, whether they be control or stratospheric heating experiments, that lower-latitude/ narrower jets exhibit longer time-scale stationary behavior, whereas higher-latitude/wider jets exhibit shorter time-scale poleward propagation. For example, in the TR2, E5 equilibrium run, the E5 heating has shifted the midlatitude jet poleward and into the regime of shorter time-scale poleward propagating variability. Moreover, in all of the experiments of Gerber et al. (2008b) and Gerber and Vallis (2007), the tropospheric situations that exhibited longer time-scale variability were those with a more equatorward midlatitude jet.

The occurrence of these two different types of variability is a common feature that has been observed in other modeling studies and, indeed, in the real atmosphere (Son and Lee 2006 and references therein). In a suite of experiments using a sGCM Son and Lee (2006) clearly demonstrated this. Many simulations were performed with varying tropical heating and high-latitude cooling. This had a similar impact on the tropospheric circulation as varying $T_{\text {ref }}$ in the experiments described here, although the heating and cooling were applied over more localized regions and, thus, did not have such a direct effect on the midlatitude temperature gradient and midlatitude baroclinicity. Son and Lee found that there were two distinct regions of the tropical heatinghigh-latitude cooling parameter space. Strong tropical heating and weak high-latitude cooling resulted in strong single jets and variability characterized by "zonal index" behavior, that is, longer time-scale fluctuations of the jet position. Conversely, weak tropical heating and strong high-latitude cooling resulted in weaker doublejet states and variability that was characterized by poleward propagation.

Lee et al. (2007) demonstrated the importance of meridional propagation of waves in producing the poleward propagating anomalies. They suggest that the difference between the states that show poleward propagation and those that exhibit the zonal index behavior lies in the structure of the meridional potential vorticity gradient of the basic state. This is discussed further by Son et al. (2008a), who suggest that it is the presence of critical or reflecting latitudes that determines whether the dominant variability is poleward propagation or zonal index behavior. This was demonstrated by calculation of a refractive index $\left(n^{2}\right)$. Where the refractive index becomes infinite a critical latitude is formed, toward which eddies are refracted and then undergo strong wave breaking. Conversely, when $n^{2}$ goes to zero, a reflecting latitude is formed, waves are reflected, and the breaking is weak. Son et al. (2008a) suggest that poleward propagation is associated with the presence of a critical latitude $\left(n^{2}=\infty\right)$, whereas zonal index behavior is associated with the presence of a reflecting latitude $\left(n^{2}=0\right)$. Calculation of the refractive index for the time mean state of the control runs of TR1 to TR5 (Fig. 10, rhs) is, however, inconsistent with this: for example, TR5 exhibits a reflecting latitude on the equatorward side of the jet, whereas it is dominated by poleward propagation, and TR1 and TR2 clearly exhibit critical latitudes $\left(n^{2} \rightarrow \infty\right)$, whereas they are characterized by the zonal index behavior.

It is clear from this study, and previous work, that changes in tropospheric jet structure affect the natural unforced variability. Furthermore, it has been shown here that different jet structures also respond differently to stratospheric heating in a manner that is, at least qualitatively, consistent with the fluctuation-dissipation theorem. Lower-latitude/narrower jets exhibit much longer time scales of variability and have a much larger magnitude of response to stratospheric heating, whereas higher-latitude/ wider jets have shorter time-scale variability and a much smaller magnitude of response to stratospheric heating. The following section investigates this by examining the time evolution of the response to stratospheric heating. 
TR1 (base=29deg)

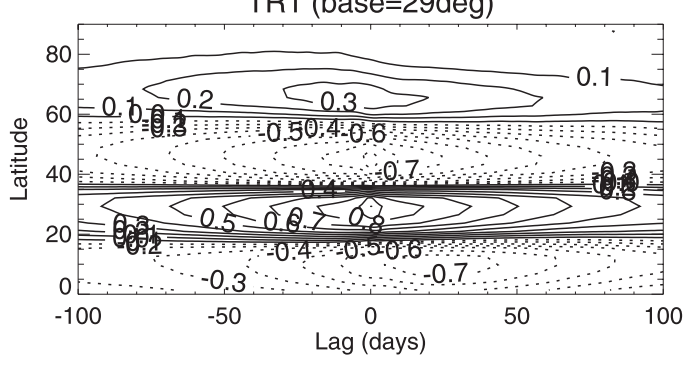

TR2 (base=34deg)

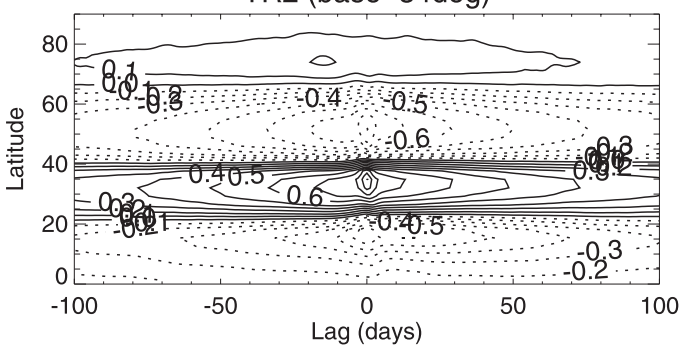

TR3 (base=38deg)

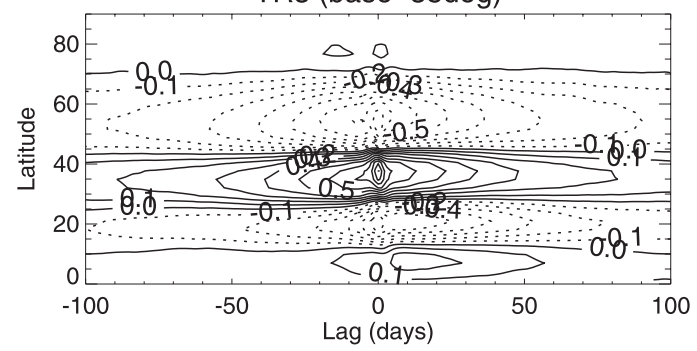

TR4 (base=40deg)

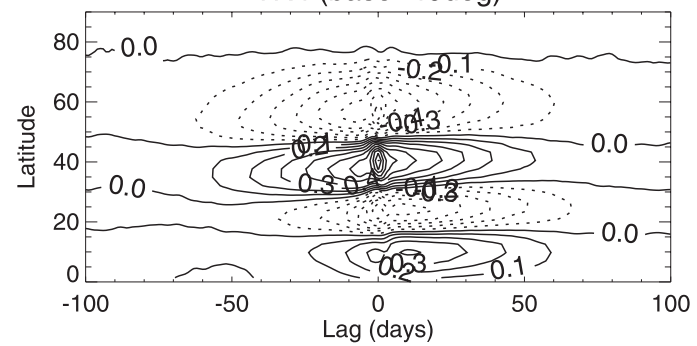

TR5 (base $=46 \mathrm{deg}$ )

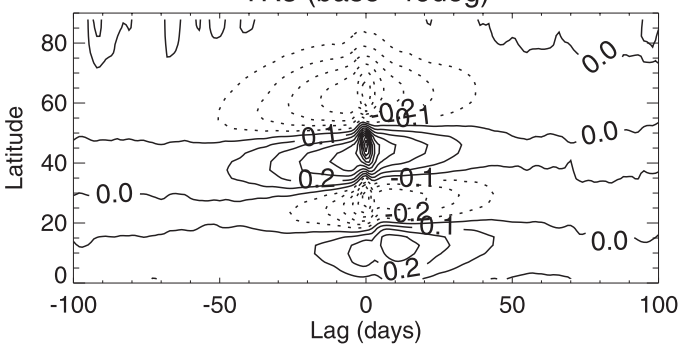

nsq TR1, 286hPa
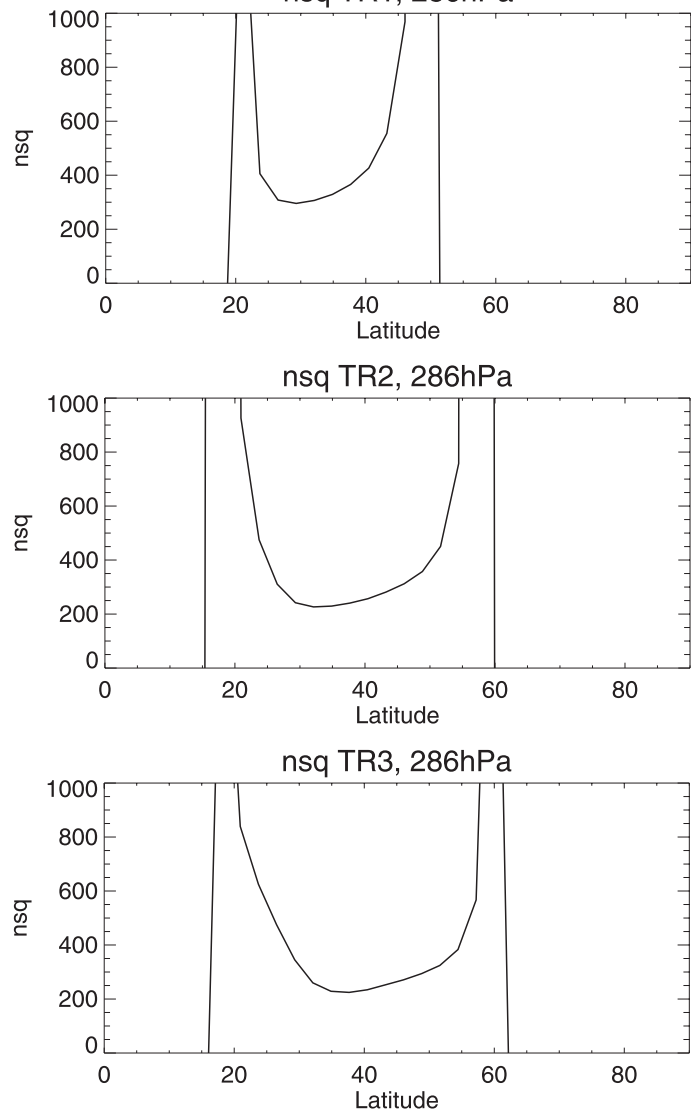

nsq TR4, 286hPa

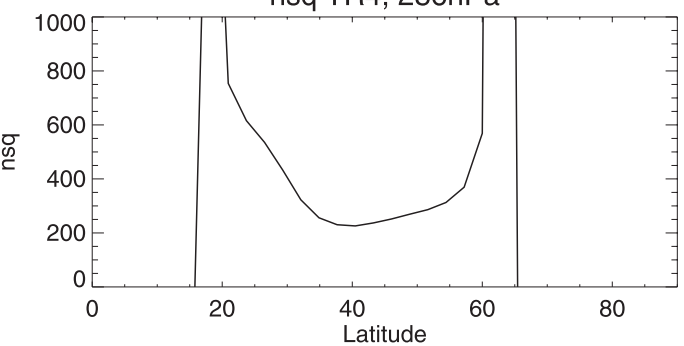

nsq TR5, 286hPa

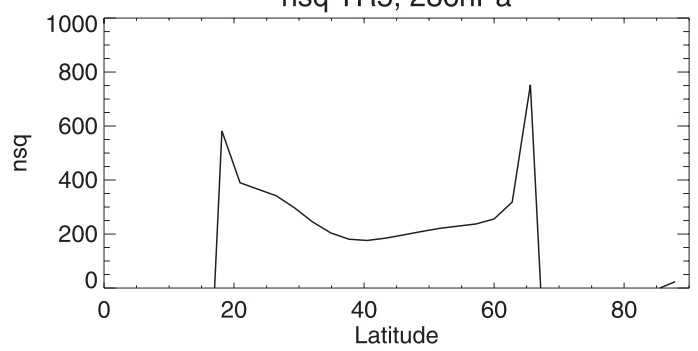

FIG. 10. (left) One-point correlation maps of the zonal-mean zonal wind anomaly as a function of latitude for the ensemble mean at the 286-hPa level for (top) TR1 to (bottom) TR5 (contour interval 0.1) and (right) refractive index at the 286-hpa level for phase speed $c=8 \mathrm{~m} \mathrm{~s}^{-1}$. 
(a) Mean lul anomaly (196hPa - surface)

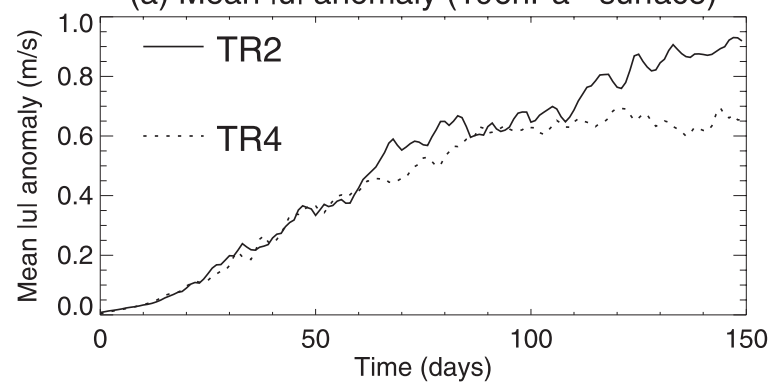

(b) Mean ITI anomaly (0 to $200 \mathrm{hPa}$ )

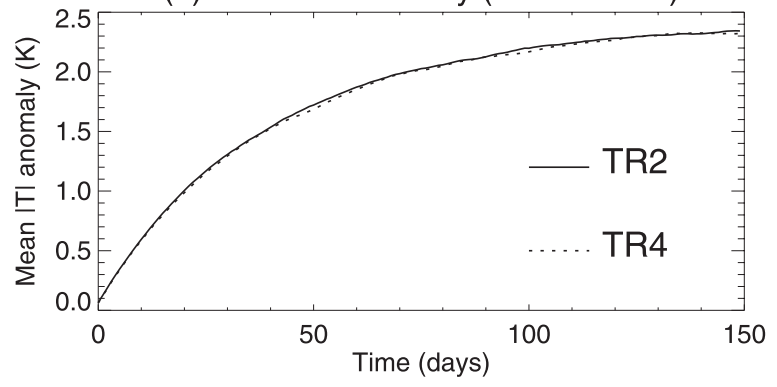

(c) Correlation (196hPa - surface)

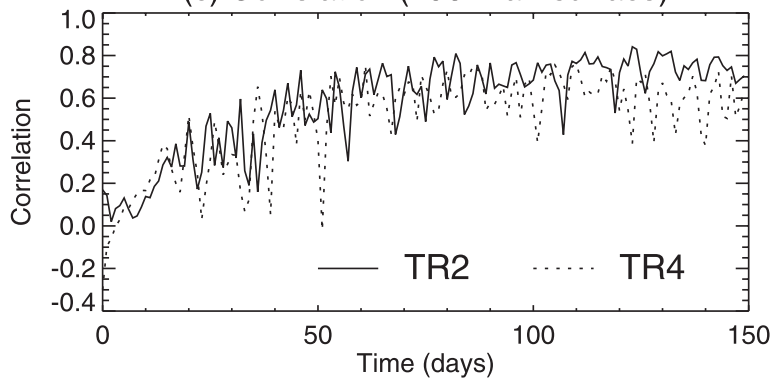

(d) Correlation (196hPa - surface), 7 day smoothing

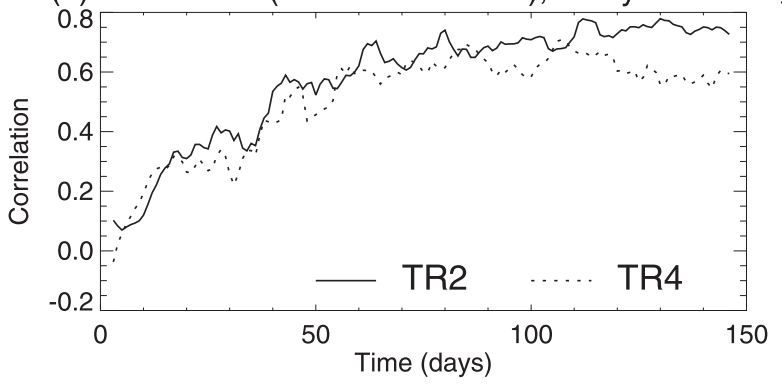

(e) Control variability correlation

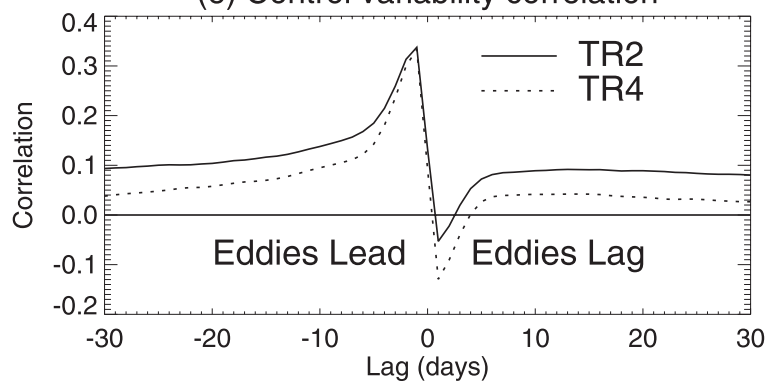

FIG. 11. (a)-(d) Spinup evolution for the E5 response in TR2 and TR4 (a) mean $|\bar{u}|$ anomaly between $196 \mathrm{hPa}$ and the surface,

\section{c. Why the difference in magnitude of response?}

Here the spinup evolution of two of the tropospheres (TR2 and TR4) is examined to investigate how the eddy feedback depends on the tropospheric control climate and how this leads to different magnitudes of equilibrated response. For each of these tropospheres, a 500-member spinup ensemble has been performed (as described in section 2c), starting from days taken from the relevant control integration and switching on the E5 stratospheric heating anomaly.

The evolution of zonal wind anomalies, eddy momentum flux, and other quantities in each of these experiments progresses in a similar manner to those in SBH09. Initially there is a weakening of the upward E-P flux around the tropopause on the equatorward flank of the jet, which reduces the equatorward wave propagation into the tropics. The resulting easterly forcing is transferred downward into the troposphere. Then the growing zonal wind anomalies increasingly refract the E-P flux equatorward across the jet center creating poleward eddy momentum flux anomalies, which act to feed back positively onto the zonal wind anomalies. As the zonal wind anomalies increase, the enhanced vertical wind shear on the poleward side of the jet increases eddy growth rates and the E-P flux divergence there and viceversa on the equatorward side of the jet.

To examine the differences between TR2 and TR4, Fig. 11 presents the evolution of selected parameters averaged over limited regions in the latitude-pressure plane. As a measure of the magnitude of the tropospheric zonal wind response, the mean absolute magnitude of the zonal wind anomaly at all latitudes and pressures between $196 \mathrm{hPa}$ and the surface has been calculated. It is clear that there is a dramatic difference in the evolution of this zonal wind metric between TR2 and TR4. Both spinups begin similarly with the wind anomaly metric increasing at a similar rate until around day 60 . However, after this the wind anomaly metric increases at a faster rate in TR2 with TR4 reaching equilibrium much earlier and at a much lower amplitude than TR2. The wind anomaly continues to increase in TR2 and does not appear to have reached equilibrium by 300 days (not shown). In contrast, the evolution of temperature in

(b) mean $|\bar{T}|$ anomaly from 0 to $200 \mathrm{hPa}$, (c) correlation between anomalies in horizontal eddy momentum flux convergence and zonal wind between $196 \mathrm{hPa}$ and the surface, and (d) as in (c) but with 7-day smoothing. (e) Same correlation diagnostic as in (c) and (d) but for the TR2 and TR4 control run variability as a function of lag between the eddy forcing and zonal wind anomalies. 
the stratosphere averaged over all latitudes between 0 and $200 \mathrm{hPa}$ (Fig. 11b) is almost identical in the two spinups. It should be noted that, as the model does not have a well-resolved stratosphere, the stratospheric temperature response is unlikely to be realistic. Rather, this is used as a diagnostic to determine how the stratospheric temperature perturbation in response to the altered $T_{\text {ref }}$ evolves over time and, thus, how the forcing on the troposphere evolves over time. So, although the time taken for the stratosphere to respond is similar in each experiment, the time over which the troposphere responds is dramatically different.

Given the mechanism presented in SBH09 and the importance of the feedback between the eddies and the mean flow, this suggests that the feedback occurs more effectively in TR2 than TR4. As a measure of the effectiveness of the tropospheric eddy momentum fluxes at feeding back onto the zonal flow anomalies, the pointby-point correlation between the eddy momentum flux convergence and the wind anomalies over all latitudes and pressures below $196 \mathrm{hPa}$ has been calculated and is presented in Figs. 11c (the daily) and 11d (7-day running mean). During the spinup a positive correlation between the eddy momentum flux convergence anomalies and the zonal wind anomalies becomes apparent as the feedback begins. After around day 60 , it is clear in both smoothed and the unsmoothed data that the correlation is consistently higher in TR2 than in TR4. This is particularly true toward the end of the spinup. Thus, the feedback involving tropospheric eddy momentum fluxes projecting back onto the zonal wind anomalies is more efficient in TR2 than in TR4. This allows the TR2 wind anomalies to keep growing longer before they reach an equilibrated state in which the anomalous eddy forcing is balanced by changes in the surface friction and the Newtonian relaxation. Exactly why this difference in strength of feedback becomes more apparent during the later part of the spinup remains unclear and is the subject of ongoing work.

To confirm that this correlation between the patterns of eddy momentum flux convergence and the zonal wind anomalies is indeed associated with a positive feedback onto the zonal wind anomalies, rather than related to some natural persistence in the eddy forcing that produces a zonal wind anomaly at any given time, Fig. 11e presents the same correlation diagnostic but for the natural control run variability of TR2 and TR4 and for various lags between the eddy momentum flux convergence and zonal wind anomalies. The results are very similar to those of Lorenz and Hartmann (2001, see their Fig. 5). The largest correlation occurs with the eddy forcing leading the zonal wind, as expected. The correlation then rapidly drops, only to increase again a few days later. This is the positive feedback onto the zonal wind anomalies found by Lorenz and Hartmann (2001). This positive feedback is larger and drops off less rapidly for TR2 than TR4, implying more persistence and a stronger feedback between the eddies and the mean flow. The correlations at lag zero are the equivalent of that plotted in Figs. 11c and 11d for the forced spinup response. The correlation at lag zero for the natural variability is small $(\sim 0.1)$ and the difference between TR2 and TR4 is barely discernable. In Figs. 11c and 11d, the correlations are considerably larger $(\sim 0.6$ and 0.7 after around day 60), as is the difference between TR2 and TR4. Therefore, the majority of this correlation apparent in the spinups comes from a positive feedback onto the forced zonal wind anomalies rather than any natural persistence in the eddy momentum flux. Furthermore, the larger correlation for TR2 than TR4 suggests that the positive feedback during the later part of the spinup is more effective for TR2.

Another mechanism whereby stratospheric temperature perturbations can result in a shift in the jet has been proposed by Chen et al. (2007) and Chen and Held (2007). By this mechanism altered vertical wind shear in response to altered meridional temperature gradients would increase the phase speed of the tropospheric eddies and shift the latitude of eddy breaking, and therefore the jet, poleward. However, it is often difficult to separate cause from effect. Is the change in phase speed causing the jet shift, or a response to it, or perhaps both? A detailed discussion of this mechanism is beyond the scope of this study as its primary purpose is to discuss the effect of varying the tropospheric basic state on the response. Preliminary analysis of eddy momentum flux cospectra following Chen et al. (2007) does indeed show an increase in phase speed in equilibrium, which is larger for the lower-latitude jets. However, in the early stages of the response in the spinup ensembles such a phase speed shift is not apparent. It therefore seems likely that the shift in phase speed is a result of and/or a feedback onto the zonal wind anomalies. A larger shift is found in, for example, TR1 compared to TR5 because the zonal wind anomalies are larger for TR1 and not because the larger phase shift in TR1 causes the larger zonal wind anomalies. This is ongoing work to be presented in a future study.

Figure 12 examines this eddy feedback correlation for the equilibrated response. It shows the mean absolute magnitude of the zonal wind anomaly over all latitudes and pressures versus the point-by-point correlation between the eddy momentum flux convergence and zonal wind anomalies in the latitude-height plane. Each of these fields is calculated from the mean of the equilibrated E5 response anomaly for each run. Figure 12a 
(a) E5, ensemble mean

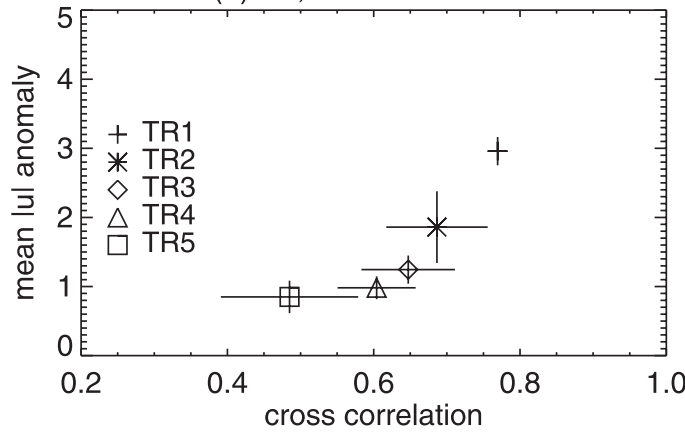

(b) E5, individual ensemble members

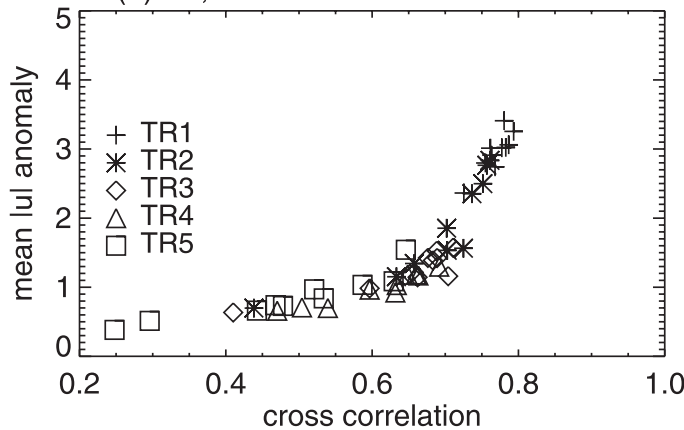

FIG. 12. (a) Mean magnitude of zonal wind anomaly vs correlation between $\overline{u^{\prime} v^{\prime}}$ convergence and zonal wind anomaly at all latitudes and pressures for the E5 runs. (b) As in (a) but for each individual ensemble member of the E5 experiments.

shows the ensemble mean and $95 \%$ confidence interval for each tropospheric state, and Fig. 12b shows each individual ensemble member. It is clear that a larger magnitude of response is accompanied by a higher correlation between the eddy momentum flux convergence and zonal wind anomalies, that is, a more efficient feedback between the eddies and the mean flow anomalies. There are large uncertainties in the ensemble mean values but nevertheless the individual ensemble members show a clearly defined trend, with the larger magnitude of response being associated with higher cross-correlation between the eddy momentum flux convergence and the zonal wind anomalies.

To conclude this section on the effect of varying tropospheric jet structure, it has been found that qualitatively similar patterns of response to stratospheric heating are produced for the different tropospheres but with dramatically different magnitudes. There is a consistent trend with lower-latitude/narrower jets having a larger magnitude of response to stratospheric heating than higher-latitude/wider jets. Examination of the control run variability for each of these tropospheres has shown that lower-latitude/narrower jets also have a much longer time scale of variability in their control run simulations. This is consistent with the idea that a feedback between the eddies and the mean flow in the troposphere is important in both producing the tropospheric response to stratospheric heating and increasing the persistence of zonal wind anomalies in the control run variability. Some aspect of the lowerlatitude/narrower jets allows the feedback between the eddies and the mean flow to be stronger, leading to both a larger magnitude zonal wind response to stratospheric heating and longer time-scale control run variability. The exact mechanism by which variations in the jet structure leads to this difference is the subject of ongoing work.

\section{Discussion and conclusions}

The effect of changing the tropospheric climatology on the response to stratospheric heating in a sGCM has been investigated. This follows on from the work of HBD05 in which it was demonstrated that heating of the lower stratosphere resulted in an equatorward or poleward shift of the tropospheric midlatitude jet, the sign of which depended on the sign and latitudinal distribution of the applied heating, and SBH09 in which a mechanism for the production of the tropospheric response was proposed.

The impact of introducing idealized topography into the model was first investigated, motivated by the study of Gerber and Vallis (2007), which showed that introducing idealized topography can substantially reduce the time scale of annular variability to more realistic values. The initial aim of this experiment was to investigate the effect of zonally asymmetric mean climate on the magnitude of response to stratospheric heating, but perhaps the more important conclusion to be drawn from it is the need for very long integrations in models such as this to accurately determine the magnitude of response. Examination of an ensemble of individual 5000-day integrations demonstrates how much variability there is in both the magnitude of response and the time scale of variability. It is clear that single integrations of several thousand days length, as commonly used with such models, are insufficient for accurate determination of the magnitude of response to a forcing and accurate characterization of the model variability. Moreover, although the decorrelation time scale is rather well constrained and of fairly short time scale in the hemisphere with topography, the magnitude of response to stratospheric heating is not well constrained and has a large variation in magnitude between different ensemble members. Thus, basing the length of integration required, for 
a given accuracy, on the time scale of control run variability (as done by Gerber et al. 2008b for examination of natural unforced variability) may not be sufficient for the accurate determination of the equilibrium response to a forcing.

The ensemble mean of the topography experiments does demonstrate a slight reduction in the magnitude of response in the presence of topography, which is consistent with a reduction in the decorrelation time scale of the control run variability in the hemisphere with topography present. This reduction in magnitude of response is small, however, compared to the magnitude of variability between the different ensemble members of the same experiment, so it is difficult to assign a value to this reduction. Nevertheless, a qualitatively similar pattern of response is found to that in HBD05 and SBH09 and, therefore, this pattern of response and the proposed mechanism appear to remain valid in the presence of zonally asymmetric tropospheric climates.

The effect of varying tropospheric jet structure was then investigated for each of five different tropospheric relaxation temperature profiles using an ensemble of ten 5000-day equilibrium E5 responses. The experiments show a dramatic influence of the structure of the tropospheric jet on the response to stratospheric heating, with lower-latitude/weaker/narrower jets having a much larger response than higher-latitude/stronger/wider jets. ${ }^{3}$ This is accompanied by a much longer time scale of variability in the control run simulations of the lowerlatitude/weaker/narrower jets, consistent with the results of Gerber and Vallis (2007) and the fluctuation-dissipation theorem. Indeed it is true that, for all experiments, the tropospheres that show a larger response to stratospheric heating tend to have longer time scale variability in their control runs. This is summarized in Fig. 13, which shows the ensemble mean projection of the zonalmean zonal wind response onto the first EOF versus the control run decorrelation time scale for each experiment. This result is consistent with the idea that it is a feedback between the eddies and the mean flow that is responsible both for maintaining the persistence of variability in the control run simulations and for amplifying the response to stratospheric heating. Moreover, it adds to a growing body of evidence (Ring and Plumb 2008; Gerber et al. 2008b) that the fluctuation dissipation theorem does work in a qualitative sense for

\footnotetext{
${ }^{3}$ It should be noted that not all lower-latitude jets are necessarily weaker. This is due to the way the experiments have been set up. Son and Lee (2006) investigate the variability of jets that are lower latitude and stronger. Moreover, the higher-latitude jets are wider simply because the eddy-driven jet is more well separated from the subtropical jet creating a wider region of westerly winds.
}

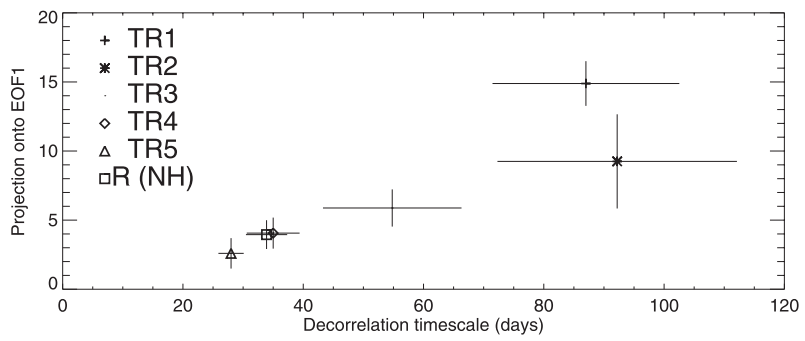

FIG. 13. Projection of zonal wind anomaly onto EOF 1 vs control run decorrelation time scale for the ensemble means of each E5 stratospheric heating experiment.

predicting the model response to a forcing on the basis of its control run variability (assuming the projection of the forcing onto EOF1 does not vary too much between experiments).

Gerber and Vallis (2007) demonstrated that the sensitivity of the decorrelation time scale to parameters such as the equator-to-pole temperature difference disappeared in the presence of topography. However, preliminary investigation suggests that the introduction of topography alters the jet structure more for lower-latitude/ narrower jets such that, in the presence of topography, lower-latitude jets are shifted into a more poleward position, whereas the position of the higher-latitude jets is affected less. Thus, the sensitivity to jet latitude is perhaps not reduced in the presence of topography but, rather, it is the difference in jet position between TR1 to TR5 that is reduced.

The model used here is highly idealized, and it remains to be demonstrated whether the relationship found here between jet latitude/width, the strength of the feedback between eddies and the mean flow, and the response to a forcing occurs in the real atmosphere. Presumably, if it does, it will be in a modified form. There is some evidence, however, for such a relationship between the latitude-width of the jet in chemistry climate models and the magnitude of the jet response to ozone depletion in the Southern Hemisphere (Son et al. 2010). Also, Kidston and Gerber (2010) have demonstrated in Coupled Model Intercomparison Project phase 3 simulations of twenty-first century climate that the magnitude of the poleward shift of the eddy-driven jet in the Southern Hemisphere associated with climate change depends on the model's climatological jet structure, with lower-latitude present-day jets having a larger future poleward shift. Possible reasons for such a relationship are discussed in that study. One possibility is that lower-latitude jets shift farther poleward in response to a forcing simply because they have farther to travel before they reach a high-latitude limit. In these simplified stratospheric heating experiments, however, a similar (although less dramatic) relationship between 
the magnitude of response to polar heating (where the jet shifts equatorward) and the latitude of the jet is found. Thus, higher-latitude jets also show a smaller magnitude of response when they are being forced equatorward.

The results of this study suggest that a more likely reason for the dependence of both the time scale of natural variability and the magnitude of response to a forcing on the structure of the jet relates to the strength of the feedback between the eddies and mean flow (Fig. 12). Recent studies by Barnes et al. (2010) and Barnes and Hartmann (2010) suggest that the presence of poleward wave breaking is important in the strength of the feedback onto annular mode variability. Lower-latitude jets tend to have critical latitudes on their poleward side and thus exhibit poleward wave breaking, whereas higher-latitude jets tend to have a reflecting latitude. They suggest that, when there is a poleward critical latitude and poleward wave breaking, the region of the eddy forcing becomes narrower and the strength of the feedback becomes stronger. However, it is difficult to see that this is true of these forced runs. Figure 8 clearly shows that the difference in strength of the feedback between low- and high-latitude jets is really related to the difference in strength of the feedback associated with equatorward propagating waves, rather than a preference for poleward wave breaking anomalies for lower-latitude jets. Some aspect of the lower-latitude/narrower jet structure results in a stronger feedback between the eddies and the mean flow, which leads to both a longer time scale of variability in the control run and a larger magnitude of response to stratospheric heating. Which aspect of the jet structure is important remains uncertain.

To conclude, it has been demonstrated that the control run variability appears to be highly dependent on model specification. Consistent with this and the ideas of the fluctuation-dissipation theorem, it has been shown that the magnitude of response to stratospheric heating is also highly dependent on the model specification. Moreover, extremely long integrations are required to accurately determine the magnitude of response to a forcing. Nevertheless, simplified GCMs are very useful tools for investigating the qualitative patterns of response to forcing and the mechanisms involved. Indeed, in all of the stratospheric heating experiments described above and in HBD05 and SBH09 the pattern of response is robust. To assess the quantitative aspects of response, however, great care must be taken to ensure that integrations of sufficient length are used and that the model is in the most realistic configuration achievable (as determined by the time scale of natural variability).

Acknowledgments. Isla Simpson was funded by a U.K. Natural Environment Research Council (NERC) Ph.D. studentship. Sarah Sparrow is funded by the NERC SOLCLI consortium grant. We are also very grateful to Fenwick Cooper for his help in the running of the ensemble simulations and to two anonymous reviewers and Ed Gerber for helpful comments.

\section{REFERENCES}

Baldwin, M. P., D. B. Stephenson, D. W. J. Thompson, T. J. Dunkerton, A. J. Charlton, and A. O'Neill, 2003: Stratospheric memory and skill of extended-range weather forecasts. Science, $\mathbf{3 0 1}$, 636-640.

- — , and I. T. Jolliffe, 2009: Spatial weighting and iterative projection methods for EOFs. J. Climate, 22, 234-243.

Barnes, E. A., and D. L. Hartmann, 2010: Testing a theory for the effect of latitude on the persistence of eddy-driven jets using CMIP3 simulations. Geophys. Res. Lett., 37, L15801, doi:10.1029/2010GL044144.

, D. M. W. Frierson, and J. Kidston, 2010: Effect of latitude on the persistence of eddy-driven jets. Geophys. Res. Lett., 37, L11804, doi:10.1029/2010GL043199.

Butler, A. H., D. W. J. Thompson, and R. Heikes, 2010: The steadystate atmospheric circulation response to climate change-like thermal forcings in a simple general circulation model. J. Climate, 23, 3474-3496.

Chan, C. J., and R. A. Plumb, 2009: The response to stratospheric forcing and its dependence on the state of the troposphere. J. Atmos. Sci., 66, 2107-2115.

Chen, G., and I. M. Held, 2007: Phase speed spectra and the recent poleward shift of Southern Hemisphere surface westerlies. Geophys. Res. Lett., 34, L21805, doi:10.1029/2007GL031200.

,-- , and W. A. Robinson, 2007: Sensitivity of the latitude of the surface westerlies to surface friction. J. Atmos. Sci., 64, 2899-2915.

Edmon, H. J., B. J. Hoskins, and M. E. Mcintyre, 1980: EliassenPalm cross sections for the troposphere. J. Atmos. Sci., 37, 2600-2616.

Gerber, E. P., and G. K. Vallis, 2007: Eddy-zonal flow interactions and the persistence of the zonal index. J. Atmos. Sci., 64, 32963311.

_ , and L. M. Polvani, 2009: Stratosphere-troposphere coupling in a relatively simple AGCM: The importance of stratospheric variability. J. Climate, 22, 1920-1933.

_ Atlantic Oscillation and annular modes. J. Atmos. Sci., 66, 332-352.

— L. M. Polvani, and D. Ancukiewicz, 2008a: Annular mode time scales in the Intergovernmental Panel on Climate Change fourth assessment report models. Geophys. Res. Lett., 35, L22707, doi:10.1029/2008GL035712.

_ S. Voronin, and L. M. Polvani, 2008b: Testing the annular mode autocorrelation time scale in simple atmospheric general circulation models. Mon. Wea. Rev., 136, 1523-1536.

Haigh, J. D., M. Blackburn, and R. Day, 2005: The response of tropospheric circulation to perturbations in lower-stratospheric temperature. J. Climate, 18, 3672-3685.

Held, I. M., and M. J. Suarez, 1994: A proposal for the intercomparison of the dynamical cores of atmospheric general circulation models. Bull. Amer. Meteor. Soc., 75, 1825-1830.

Hoskins, B. J., and A. J. Simmons, 1975: Multilayer spectral model and semi-implicit method. Quart. J. Roy. Meteor. Soc., 101, $637-655$. 
Kidston, J., and E. P. Gerber, 2010: Intermodel variability of the poleward shift of the austral jet stream in the CMIP3 integrations linked to biases in 20th-century climatology. Geophys. Res. Lett., 37, L09708, doi:10.1029/2010GL042873.

Kushner, P. J., and L. M. Polvani, 2004: Stratosphere-troposphere coupling in a relatively simple AGCM: The role of eddies. J. Climate, 17, 629-639.

— atively simple AGCM: Impact of the seasonal cycle. J. Climate, 19, 5721-5727.

Lee, S., S. W. Son, K. Grise, and S. B. Feldstein, 2007: A mechanism for the poleward propagation of zonal mean flow anomalies. J. Atmos. Sci., 64, 849-868.

Leith, C. E., 1975: Climate response and fluctuation dissipation. J. Atmos. Sci., 32, 2022-2026.

Lorenz, D. J., and D. L. Hartmann, 2001: Eddy-zonal flow feedback in the Southern Hemisphere. J. Atmos. Sci., 58, 33123327.

— , and E. T. DeWeaver, 2007: Tropopause height and zonal wind response to global warming in the IPCC scenario integrations. J. Geophys. Res., 112, D10119, doi:10.1029/2006JD008087.

Polvani, L. M., and P. J. Kushner, 2002: Tropospheric response to stratospheric perturbations in a relatively simple general circulation model. Geophys. Res. Lett., 29, 1114, doi:10.1029/ 2001 GL014284.

Ring, M. J., and R. A. Plumb, 2008: The response of a simplified GCM to axisymmetric forcings: Applicability of the fluctuationdissipation theorem. J. Atmos. Sci., 65, 3880-3898.

Robinson, W. A., 2000: A baroclinic mechanism for the eddy feedback on the zonal index. J. Atmos. Sci., 57, 415-422.
Simmons, A. J., and D. M. Burridge, 1981: An energy and angular momentum conserving vertical finite-difference scheme and hybrid vertical coordinates. Mon. Wea. Rev., 109, 758-766.

Simpson, I. R., M. Blackburn, and J. D. Haigh, 2009: The role of eddies in driving the tropospheric response to stratospheric heating perturbations. J. Atmos. Sci., 66, 1347-1365.

Son, S. W., and S. Lee, 2006: Preferred modes of variability and their relationship with climate change. J. Climate, 19, 2063-2075.

,-- S. B. Feldstein, and J. E. T. Hoeve, 2008a: Time scale and feedback of zonal mean flow variability. J. Atmos. Sci., 65, 935-952.

— , and Coauthors, 2008b: The impact of stratospheric ozone recovery on the Southern Hemisphere westerly jet. Science, 320, $1486-1489$.

— , and Coauthors, 2010: The impact of stratospheric ozone on Southern Hemisphere circulation change: A multimodel assessment. J. Geophys. Res., 115, D00M07, doi:10.1029/2010JD014271.

Song, Y. C., and W. A. Robinson, 2004: Dynamical mechanisms for stratospheric influences on the troposphere. J. Atmos. Sci., 61, $1711-1725$

Sparrow, S. N., M. Blackburn, and J. D. Haigh, 2009: Annular variability and eddy-zonal flow interactions in a simplified atmospheric GCM. Part I: Characterization of high- and lowfrequency behavior. J. Atmos. Sci., 66, 3075-3094.

Williams, G. P., 2006: Circulation sensitivity to tropopause height. J. Atmos. Sci., 63, 1954-1961.

Wittman, M. A. H., L. M. Polvani, R. K. Scott, and A. J. Charlton, 2004: Stratospheric influence on baroclinic lifecycles and its connection to the arctic oscillation. Geophys. Res. Lett., 31, L16113, doi:10.1029/2004GL020503. 